\title{
MULTISCALE ANALYSIS OF NUTRIENT UPTAKE BY PLANT ROOTS WITH SPARSE DISTRIBUTION OF ROOT HAIRS: NONSTANDARD SCALING*
}

\author{
JOHN R. KING ${ }^{\dagger}$, JAKUB KÖRY ${ }^{\ddagger}$, AND MARIYA PTASHNYK ${ }^{\S}$
}

\begin{abstract}
In this paper we undertake a multiscale analysis of nutrient uptake by plant roots by considering different scale relations between the radius of root hairs and the distance between them. We combine the method of formal asymptotic expansions and rigorous derivation of macroscopic equations. The former prompt us to study a distinguished limit (which yields a distinct effective equation), allow us to determine higher-order correctors, and provide motivation for the construction of correctors essential for rigorous derivation of macroscopic equations. The results of our asymptotic analysis are validated by direct comparison with full-geometry numerical simulations.
\end{abstract}

Key words. homogenization, parabolic equations, nutrient uptake by plants, sparse root hairs, nonstandard scaling, domains perforated by very thin tubes

AMS subject classifications. 35Bxx, 35K20, 35Q92, 35K60, 92C 80

DOI. $10.1137 / 19 \mathrm{M} 1309626$

1. Introduction. An efficient nutrient uptake by plant roots is very important for plant growth and development $[2,4]$. Root hairs, the cylindrically shaped lateral extensions of epidermal cells that increase the surface area of the root system, play a significant role in the uptake of nutrients by plant roots [10]. Thus to optimize the nutrient uptake it is important to understand the impact of root hairs on the uptake processes. Early phenomenological models describe the effect of root hairs on the nutrient uptake by increasing the radius of roots [28]. Microscopic modeling and analysis of nutrient uptake by root hairs on the scale of a single hair, assuming periodic distribution of hairs and that the distance between them is of the same order as their radius, were considered in [20, 29,33].

In contrast to previous results, in this work we consider a sparse distribution of root hairs, with the radius of root hairs much smaller than the distance between them. We consider two different regimes given by scaling relations between the hair radius and the distance between neighboring hairs. Applying multiscale analysis techniques, we derive macroscopic equations from the microscopic description by applying both the method of formal asymptotic expansions and rigorous proofs of convergences of sequences of solutions of microscopic (full-geometry) problems. Due to nonstandard scale relations between the size of the microscopic structure and the periodicity, the homogenization techniques of two-scale convergence, the periodic unfolding method, $\Gamma$ - or G-convergences (see, e.g., [13, 24, 25, 27]) do not apply directly, and a different approach needs to be developed. The construction of inner and outer layer approxima-

\footnotetext{
* Received by the editors December 30, 2019; accepted for publication (in revised form) March 30, 2021; published electronically July 12, 2021.

https://doi.org/10.1137/19M1309626

Funding: JRK and JK were supported by FUTUREROOTS Project (project ID 294729) between the European Research Council and the University of Nottingham.

${ }^{\dagger}$ School of Mathematical Sciences and Centre for Plant Integrative Biology, School of Biosciences, University of Nottingham, Nottingham NG7 2RD, UK (john.king@nottingham.ac.uk).

${ }^{\ddagger}$ School of Mathematics and Statistics, University of Glasgow, University Place, Glasgow G12 8QQ, UK (Jakub.Koery@glasgow.ac.uk).

§School of Mathematical and Computer Sciences, Heriot-Watt University, Edinburgh EH14 4AL, UK (m.ptashnyk@hw.ac.uk).
} 
tion problems constitutes the main idea in the derivation of the macroscopic problems using formal asymptotic expansions. This approach allows us also to obtain equations for higher-order approximations to the macroscopic solutions. To show convergence of solutions of the multiscale (microscopic) problems to those of the corresponding macroscopic problems, we construct appropriate correctors to pass to the limit in the integrals over the boundaries of the microstructure given by root hairs. We also compare numerical solutions of the multiscale problems with solutions of macroscopic problems and higher- (first- and second-) order approximations, derived for different scale relations between the size of the hairs and the size of the periodicity.

Similar results for elliptic equations and variational inequalities were obtained in $[14,15,16]$ using the monotonicity of the nonlinear function in the boundary conditions and a variational inequality approach. The construction of correctors near surfaces of very small holes was considered in $[6,9]$ to derive macroscopic equations for linear elliptic problems with zero Dirichlet and given Robin boundary conditions. The extension of the periodic unfolding method to domains with very small holes was introduced in [5] to analyze linear wave and heat equations posed in periodically perforated domains with small holes and Dirichlet conditions on the boundary of the holes.

The paper is organized as follows. In section 2 we formulate a model for nutrient uptake by plant roots and root hairs. In section 3 we derive macroscopic equations and equations for the first- and second-order correctors, for different scale relations between the radius of root hairs and the distance between them, by using formal asymptotic expansions. The proof of the convergence of a sequence of solutions of the multiscale problem to those of the macroscopic equations via the construction of corresponding microscopic correctors is given in section 4 . The linear and nonlinear Robin boundary conditions depending on solution of the microscopic problem considered in this article require new ideas in the construction of the corresponding correctors. Numerical simulations of both multiscale and macroscopic problems are presented in section 5 , and we conclude in section 6 with a brief discussion.

2. Formulation of the problem. We consider diffusion of nutrients in a domain around a plant root and its uptake by root hairs and through the root surface. The representative length of the root is chosen to be $R=1 \mathrm{~cm}$, and the model is subsequently formulated in dimensionless terms (see the supplementary material [local/web $315 \mathrm{~KB}]$ for comments on the nondimensionalization and on parameter values). The root surface is treated as planar, which approximates the actual (curved) geometry well enough, provided that the distance between hairs measured at the root surface is comparable to the distance between hair tips, as discussed in [20]. A generalization that addresses root curvature is investigated in [18].

Consider a domain $\Omega=G \times(0, M)$ around a single plant root, with $M>0$ being representative of the half-distance between neighboring roots, where the Lipschitz domain $G \subset \mathbb{R}^{2}$ represents the part of the root surface under consideration. We assume that the root hairs are circular cylinders (of dimensionless length $L$, with $L<M$, and radius $r_{\varepsilon}$ ) orthogonal to the (planar) root surface on which they are periodically distributed; see Figure 1(a). A single root hair can be described as

$$
B_{r_{\varepsilon}} \times(0, L), \quad \text { where } B_{r_{\varepsilon}}=\left\{\left(x_{1}, x_{2}\right) \in \mathbb{R}^{2}: x_{1}^{2}+x_{2}^{2}<r_{\varepsilon}^{2}\right\} .
$$

Denoting by $Y=(-1 / 2,1 / 2)^{2}$ the unit cell and taking $\varepsilon$ to be the small parameter (the representative distance between the root hairs, which is small compared to the 


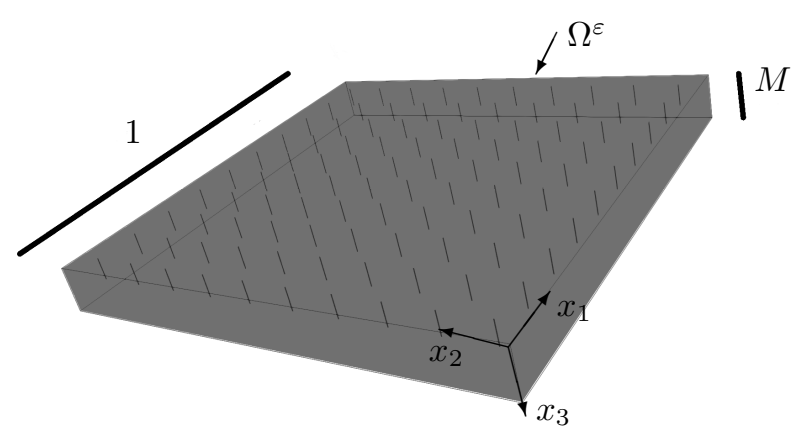

(a) Multiscale domain

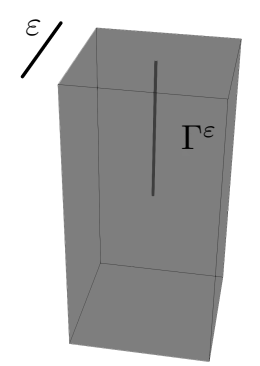

(b) Periodicity cell

FIG. 1. Problem geometry.

root length), the set of root hairs belonging to the root surface can be written as

$$
\Omega_{1, L}^{\varepsilon}=\bigcup_{\xi \in \Xi^{\varepsilon}}\left(\bar{B}_{r_{\varepsilon}}+\varepsilon \xi\right) \times(0, L), \quad \text { with } \Xi^{\varepsilon}=\left\{\xi \in \mathbb{Z}^{2}: \varepsilon(Y+\xi) \subset G\right\},
$$

i.e., we only include the root hairs whose base is fully contained in $G$. The solution domain is then defined by $\Omega^{\varepsilon}=\Omega \backslash \bar{\Omega}_{1, L}^{\varepsilon}$.

We assume the root hairs are sparsely distributed, i.e., $r_{\varepsilon} \ll \varepsilon \ll 1$, and we define $a_{\varepsilon}=r_{\varepsilon} / \varepsilon \ll 1$, and assume that $M=O(1)$ and $L=O(1)$. The surfaces of the root hairs are given by

$$
\Gamma^{\varepsilon}=\bigcup_{\xi \in \Xi^{\varepsilon}}\left(\partial B_{r_{\varepsilon}}+\varepsilon \xi\right) \times(0, L) .
$$

We shall also use the notation $\Omega_{L}=G \times(0, L)$ corresponding to the range of $x_{3}$ occupied by root hairs.

Outside the root hairs we consider the diffusion of nutrients

$$
\partial_{t} u_{\varepsilon}=\nabla \cdot\left(D_{u} \nabla u_{\varepsilon}\right) \quad \text { in } \quad \Omega^{\varepsilon}, t>0,
$$

with constant (dimensionless) diffusion coefficient $D_{u}>0$, and assume that nutrients are taken up on the root surface according to

$$
D_{u} \nabla u_{\varepsilon} \cdot \mathbf{n}=-\beta u_{\varepsilon} \quad \text { on } \quad \Gamma_{R}^{\varepsilon}, t>0,
$$

where $\Gamma_{R}^{\varepsilon}=\overline{\Omega^{\varepsilon}} \cap\left\{x_{3}=0\right\}$ defines the surface of the root (excluding the root hairs) ${ }^{1}$ and on the surfaces of the root hairs

$$
D_{u} \nabla u_{\varepsilon} \cdot \mathbf{n}=-\varepsilon K\left(a_{\varepsilon}\right) g\left(u_{\varepsilon}\right) \quad \text { on } \quad \Gamma^{\varepsilon}, t>0,
$$

where $\mathbf{n}$ denotes the outer-pointing unit normal vector to $\partial \Omega^{\varepsilon}, \beta \geq 0$ is an uptake rate, $g(\eta)$ is smooth (continuously differentiable) and monotone nondecreasing for $\eta \in[-\tilde{\varsigma}, \infty)$, with some $\tilde{\varsigma}>0$, and $g(\eta)=g_{1}(\eta)+g_{2}(\eta)$, where $g_{1}(\eta) \geq 0$ for $\eta \geq 0$, with $g_{1}(0)=0$, and $g_{2}$ is sublinear, with $g_{2}(0) \leq 0$. The monotonicity of $g$ ensures

\footnotetext{
${ }^{1}$ Even though the analysis for a nonlinear boundary condition would be straightforward, we consider linear uptake here, as the emphasis will be on the derivation of sink terms resulting from the boundary conditions applied on the hair surfaces, which are often dominant in nutrient uptake.
} 
existence of a unique solution $h$ of $h+\sigma g(h)=\zeta$, with $\zeta \geq 0$ and $\sigma>0$, important for the derivation of macroscopic equations for $(2.1)-(2.3),(2.6),(2.7)$. In section 5 we will consider the Michaelis-Menten type function

$$
g(u)=\frac{u}{1+u},
$$

often used in modeling uptake processes by plant roots (see, e.g., $[8,11])$, for which all the above assumptions are satisfied, with $g_{2} \equiv 0$. The scaling factor $K\left(a_{\varepsilon}\right)$ in $(2.3)$ is set to be

$$
K\left(a_{\varepsilon}\right)=\frac{\kappa}{a_{\varepsilon}}
$$

with some positive constant $\kappa=O(1)$ (see the supplementary material [local/web $315 \mathrm{~KB}]$ for justification of this scaling). On other parts of the boundary $\partial \Omega^{\varepsilon}$ we consider

$$
D_{u} \nabla u_{\varepsilon} \cdot \mathbf{n}=0 \quad \text { on } \quad \partial \Omega^{\varepsilon} \backslash\left(\Gamma^{\varepsilon} \cup \Gamma_{R}^{\varepsilon}\right), \quad t>0 .
$$

The initial nutrient concentration is given by

$$
u_{\varepsilon}(0, x)=u_{\text {in }}(x) \quad \text { for } x \in \Omega^{\varepsilon},
$$

where we assume that $u_{\text {in }} \in H^{2}(\Omega)$ and $0 \leq u_{\text {in }}(x) \leq u_{\max }$ for $x \in \Omega$.

First, we consider the definition of a weak solution of (2.1)-(2.3), (2.6), and (2.7). We shall use the notation $\Omega_{T}^{\varepsilon}=(0, T) \times \Omega^{\varepsilon}, \Gamma_{T}^{\varepsilon}=(0, T) \times \Gamma^{\varepsilon}$, and $\Gamma_{R, T}^{\varepsilon}=(0, T) \times \Gamma_{R}^{\varepsilon}$.

DeFinition 2.1. A weak solution of problem (2.1)-(2.3), (2.6), (2.7) is a function $u_{\varepsilon} \in L^{2}\left(0, T ; H^{1}\left(\Omega^{\varepsilon}\right)\right)$, with $\partial_{t} u_{\varepsilon} \in L^{2}\left((0, T) \times \Omega^{\varepsilon}\right)$, satisfying

$$
\int_{\Omega_{T}^{\varepsilon}}\left(\partial_{t} u_{\varepsilon} \phi+D_{u} \nabla u_{\varepsilon} \cdot \nabla \phi\right) d x d t=-\varepsilon \int_{\Gamma_{T}^{\varepsilon}} \frac{\kappa}{a_{\varepsilon}} g\left(u_{\varepsilon}\right) \phi d \gamma^{\varepsilon} d t-\int_{\Gamma_{R, T}^{\varepsilon}} \beta u_{\varepsilon} \phi d \gamma^{\varepsilon} d t
$$

for $\phi \in L^{2}\left(0, T ; H^{1}\left(\Omega^{\varepsilon}\right)\right)$ and $u_{\varepsilon}(t) \rightarrow u_{\text {in }}$ in $L^{2}\left(\Omega^{\varepsilon}\right)$ as $t \rightarrow 0$.

Standard results for parabolic equations, together with the above assumptions on $g$, ensure the existence of a unique weak solution of problem (2.1)-(2.3), (2.6), (2.7) for any fixed $\varepsilon>0$; see, e.g., [19, 21].

3. Derivation of the macroscopic equations using the method of formal asymptotic expansions. To derive the macroscopic equations from the multiscale problem (2.1)-(2.3), (2.6), (2.7) we first apply the method of the formal asymptotic expansions. We shall consider different scalings for $a_{\varepsilon}$ and derive equations for zero, first, and second orders of approximation for solutions. Apart from the macroscopic variables $x=\left(x_{1}, x_{2}, x_{3}\right)$, we further introduce $y=\left(y_{1}, y_{2}\right)=\left(x_{1} / \varepsilon, x_{2} / \varepsilon\right)$ and $z=$ $\left(z_{1}, z_{2}\right)=\left(x_{1} / r_{\varepsilon}, x_{2} / r_{\varepsilon}\right)=\left(y_{1} / a_{\varepsilon}, y_{2} / a_{\varepsilon}\right)$. Since there is no microscopic variation in the $x_{3}$ direction, we do not include any dependence on $y_{3}$ (or $z_{3}$ ). Notice that due to the assumed scale separation between the radius of the root hairs and the distance between them, three scales are present: an inner microscopic scale, $\|z\|=$ $\sqrt{z_{1}^{2}+z_{2}^{2}}=O(1)$, corresponding to the radius of root hairs; an outer microscopic scale, $\|y\|=O(1)$, given by the distance between them; and a macroscopic scale, $\|x\|=O(1)$, corresponding to a representative length of a plant root (for simplicity, we assume that the typical distance between two neighboring roots is of the same order as the representative root length). 
In the derivation of macroscopic equations we consider two cases. In the first, we take the limits in the order $\varepsilon \rightarrow 0$ and then $a_{\varepsilon} \rightarrow 0$, with no relationship assumed between these two parameters; in the second, we study a distinguished limit motivated by the analysis in section 3.1. Note that in the first case, instead of $a_{\varepsilon}$, we suppress the subscript to recall that $a$ and $\varepsilon$ are independent small parameters therein.

3.1. Derivation of the macroscopic equations in the case of complete scale separation between $\varepsilon$ and $\boldsymbol{a}$. In this section, we assume complete scale separation between $\varepsilon$ and $a$ (i.e., we take the limit $\varepsilon \rightarrow 0$ followed by $a \rightarrow 0$ ). We adopt the ansatz

$$
u_{\varepsilon}(t, x, a)=u_{0}(t, x, \hat{x} / \varepsilon, a)+\varepsilon u_{1}(t, x, \hat{x} / \varepsilon, a)+\varepsilon^{2} u_{2}(t, x, \hat{x} / \varepsilon, a)+\cdots
$$

for $x \in \Omega_{L}, t>0, \hat{x}=\left(x_{1}, x_{2}\right)$, and $u_{j}(t, x, \cdot, a)$ are assumed to be $Y$-periodic (cf. $[3,17])$. We first fix $0<a<1 / 2$ and then perform a separate $a \rightarrow 0$ analysis at each order in $\varepsilon$. Note that for the simplicity of presentation, we will consider a linear boundary condition in (2.3), i.e., $g(u)=u$; the same calculations have also been performed for a nonlinear function $g(u)$ using Taylor expansion of $g(u)$ about $u_{0}$ (see the supplementary material [local/web $315 \mathrm{~KB}]$ ).

3.1.1. $\boldsymbol{a}=\boldsymbol{O}(\mathbf{1})$. Even though this problem has already been analyzed in $[20$, 29], to set up for the sublimit $a \rightarrow 0$ in the next section, we briefly recall the main outcomes of this analysis. The terms of order $\varepsilon^{-2}$ in (2.1) and of order $\varepsilon^{-1}$ in (2.3) yield

$$
\nabla_{y} \cdot\left(D_{u} \nabla_{y} u_{0}\right)=0 \quad \text { in } Y_{a}, \quad D_{u} \nabla_{y} u_{0} \cdot \hat{\mathbf{n}}=0 \quad \text { on } \Gamma_{a}, \quad u_{0} \text { is } Y \text {-periodic, }
$$

where $Y_{a}=Y \backslash \bar{B}_{a}, \Gamma_{a}=\partial B_{a}$. The existence and uniqueness theory for linear elliptic equations with zero-flux and periodic boundary conditions implies that solutions of (3.2) are independent of $y$, i.e., $u_{0}=u_{0}(t, x, a)$. For the terms of order $\varepsilon^{-1}$ in (2.1) and of order $\varepsilon^{0}$ in (2.3) we then have

$$
\nabla_{y} \cdot\left(D_{u} \nabla_{y} u_{1}\right)=0 \quad \text { in } Y_{a}, \quad D_{u} \nabla_{y} u_{1} \cdot \hat{\mathbf{n}}=-D_{u} \nabla_{\hat{x}} u_{0} \cdot \hat{\mathbf{n}} \quad \text { on } \Gamma_{a},
$$

and $u_{1}$ is $Y$-periodic, where $\hat{x}=\left(x_{1}, x_{2}\right)$. The solution reads

$$
u_{1}(t, x, y, a)=U_{1}(t, x, a)+\nabla_{\hat{x}} u_{0}(t, x, a) \cdot \boldsymbol{\nu}(y, a),
$$

where $U_{1}$ consists of contributions to $u_{1}$ that do not depend on the microscale, and the vector function $\boldsymbol{\nu}(y, a)=\left(\nu_{1}(y, a), \nu_{2}(y, a)\right)$ is a solution of

$$
\nabla_{y} \cdot\left(D_{u} \nabla_{y} \boldsymbol{\nu}\right)=0 \quad \text { in } Y_{a}, \quad \nabla_{y} \boldsymbol{\nu} \cdot \hat{\mathbf{n}}=-\hat{\mathbf{n}} \quad \text { on } \Gamma_{a}, \quad \boldsymbol{\nu} \text { is } Y \text {-periodic. }
$$

Finally, collecting the terms of order $\varepsilon^{0}$ in (2.1) and of order $\varepsilon$ in (2.3) yields

$$
\begin{array}{lll}
\nabla_{y} \cdot\left(D_{u} \nabla_{y} u_{2}\right)=\partial_{t} u_{0}-\nabla_{x} \cdot\left(D_{u} \nabla_{x} u_{0}\right)-\nabla_{\hat{x}} \cdot\left(D_{u} \nabla_{y} u_{1}\right)-\nabla_{y} \cdot\left(D_{u} \nabla_{\hat{x}} u_{1}\right) & \text { in } Y_{a}, \\
(3.6) & D_{u} \nabla_{y} u_{2} \cdot \hat{\mathbf{n}}=-K(a) u_{0}-D_{u} \nabla_{\hat{x}} u_{1} \cdot \hat{\mathbf{n}} & \text { on } \Gamma_{a} .
\end{array}
$$

Integrating (3.6) over $Y_{a}$ and using the divergence theorem (for more details see [18]) gives as the leading-order macroscale problem

$$
\partial_{t} u_{0}=\nabla_{x} \cdot\left(D_{u} \boldsymbol{D}_{\text {eff }}(a) \nabla_{x} u_{0}\right)-\frac{2 \pi a K(a)}{1-\pi a^{2}} u_{0},
$$

where $\boldsymbol{D}_{\text {eff }}(a)=\boldsymbol{I}+\boldsymbol{B}(a) /\left(1-\pi a^{2}\right), \boldsymbol{I}$ is the identity matrix, and

$$
\boldsymbol{B}(a)=\left(\begin{array}{ccc}
\int_{Y_{a}} \frac{\partial \nu_{1}(y, a)}{\partial y_{1}} d y & 0 & 0 \\
0 & \int_{Y_{a}} \frac{\partial \nu_{2}(y, a)}{\partial y_{2}} d y & 0 \\
0 & 0 & 0
\end{array}\right) .
$$


3.1.2. $a \ll 1$. Now, we analyze (3.5) and (3.7) in the limit $a \rightarrow 0$. Because of the large scale difference between the periodicity of the microscopic structure and the radius of the root hairs, in the analysis of the asymptotic behavior of the solution we can distinguish between the behavior in a region characterized by $\|z\|=O(1)$, which will correspond to an inner solution (denoted using a superscript $I$ ), and the behavior in a region characterized by $\|y\|=O(1)$, corresponding to an outer solution (denoted using a superscript $O$ ); see [18] for more details. Thus each term in (3.1) requires its inner and outer analyses, some of which will involve expanding in $\delta=$ $1 / \ln \left(a^{-1}\right) \ll 1$. These logarithmic relationships arise due to the two-dimensional microstructure, reflecting the fact that the Green function of the Laplace operator in $\mathbb{R}^{2}$ is proportional to $\ln (r)$, as will become obvious at $O\left(\varepsilon^{2}\right)$. Note that for any $n \geq 2$, we have

$\cdots \ll \varepsilon^{n} \ll \cdots \ll \varepsilon \ll \cdots \ll a^{n} \ll \cdots \ll a \ll \cdots \ll \delta^{n} \ll \cdots \ll \delta=1 / \ln \left(a^{-1}\right) \ll 1$,

due to the assumption of the complete scale separation between $a$ and $\varepsilon$. We expand

$$
u_{0}(t, x, \delta)=u_{0,0}(t, x)+o(1) .
$$

The macroscopic behavior of $u_{0,0}$ will be determined via the Fredholm alternative at $O\left(\varepsilon^{2}\right)$ (see (3.23)). Proceeding to $O(\varepsilon)$, we should not aim to satisfy the boundary condition from (3.5) on $\Gamma_{a}$ in the $\|y\|=O(1)$ region (this part of the boundary degenerates to a point in the limit $a \rightarrow 0$ ), and we have an expansion

$$
\boldsymbol{\nu}^{O}(y, a)=\boldsymbol{\nu}_{0}^{O}(y)+a \boldsymbol{\nu}_{1}^{O}(y)+\cdots,
$$

with $\boldsymbol{\nu}_{i}^{O}$ being $Y$-periodic and satisfying Laplace's equation. Setting $z=y / a$ in (3.5) yields

$$
\nabla_{z} \cdot\left(D_{u} \nabla_{z} \boldsymbol{\nu}\right)=0 \quad \text { in } Y_{1 / a}, \quad \nabla_{z} \boldsymbol{\nu} \cdot \hat{\mathbf{n}}=-a \hat{\mathbf{n}} \quad \text { on } \partial B_{1},
$$

where $Y_{1 / a}=a^{-1} Y \backslash \bar{B}_{1}$. This suggests an inner expansion of the form

$$
\boldsymbol{\nu}^{I}(z, a)=\boldsymbol{\nu}_{0}^{I}(z)+a \boldsymbol{\nu}_{1}^{I}(z)+\cdots .
$$

It follows that $\boldsymbol{\nu}_{0}^{I}$ is independent of $z$ and that

$$
\boldsymbol{\nu}_{1}^{I}(z)=-\left[\alpha\left(r+\frac{1}{r}\right)+r\right] \frac{\left(z_{1}, z_{2}\right)}{r},
$$

where $r=\|z\|$, and $\alpha=-1$ is required to match the outer region. Hence

$$
\boldsymbol{\nu}_{1}^{I}(z)=\frac{\left(z_{1}, z_{2}\right)}{\|z\|^{2}} .
$$

To match the inner $\boldsymbol{\nu}^{I}$ and outer $\boldsymbol{\nu}^{O},(3.10)$ has to contain terms of the form

$$
a \frac{\left(z_{1}, z_{2}\right)}{\|z\|^{2}}=a^{2} \frac{\left(y_{1}, y_{2}\right)}{\|y\|^{2}}
$$

as $\|y\| \rightarrow 0$. Noting that the solution of

$$
\Delta_{y} \boldsymbol{v}(y)=2 \pi \nabla_{y} \delta(y), \quad \boldsymbol{v} \text { is } Y \text {-periodic, }
$$


where $\delta(y)$ is the Dirac delta, has the behavior

$$
\boldsymbol{v}(y) \sim \frac{\left(y_{1}, y_{2}\right)^{T}}{\|y\|^{2}} \quad \text { as }\|y\| \rightarrow 0,
$$

we infer that $\boldsymbol{\nu}_{2}^{O}=\boldsymbol{v}$. In order to uncover the effective behavior at the macroscale, we need to analyze (3.6) in the inner and outer regions, and matching between these will eventually lead us to the homogenized equation (3.23). Using the information on the inner and outer behaviors of $u_{1}$ (see (3.4) and (3.14)), problem (3.6) becomes

$$
\begin{aligned}
\nabla_{y} \cdot\left(D_{u} \nabla_{y} u_{2}\right) & =\partial_{t} u_{0}-\nabla_{x} \cdot\left(D_{u} \nabla_{x} u_{0}\right)+O(a) & & \text { in } Y_{a}, \\
D_{u} \nabla_{y} u_{2} \cdot \hat{\mathbf{n}} & =-K(a) u_{0}-D_{u} \nabla_{\hat{x}}\left(U_{1}+\nabla_{\hat{x}} u_{0} \cdot \boldsymbol{\nu}\right) \cdot \hat{\mathbf{n}} & & \text { on } \Gamma_{a} .
\end{aligned}
$$

Rescaling by $z=y / a$ and using (2.5), we obtain

$$
\begin{aligned}
\nabla_{z} \cdot\left(D_{u} \nabla_{z} u_{2}\right) & =O\left(a^{2}\right) & & \text { in } Y_{1 / a}, \\
D_{u} \nabla_{z} u_{2} \cdot \hat{\mathbf{n}} & =-\kappa u_{0}+O(a) & & \text { on } \partial B_{1},
\end{aligned}
$$

Recalling (3.9), we infer the ansatz for $u_{2}$,

$$
u_{2}(t, x, y, \delta)=U_{2}(t, x, \delta)+u_{0}(t, x, \delta) \psi(y, \delta),
$$

where the inner $(z=y / a=O(1))$ expansion for $\psi$ reads

$$
\psi^{I}(z, \delta)=\psi_{0}^{I}(z)+O(\delta),
$$

and at the leading order we get

$$
\nabla_{z} \cdot\left(D_{u} \nabla_{z} \psi_{0}^{I}\right)=0 \quad \text { in } Y_{\infty}, \quad D_{u} \nabla_{z} \psi_{0}^{I} \cdot \hat{\mathbf{n}}=-\kappa \quad \text { on } \partial B_{1},
$$

where $Y_{\infty}=\mathbb{R}^{2} \backslash \bar{B}_{1}$, the solution of which reads

$$
\psi_{0}^{I}(z)=\left(\kappa / D_{u}\right) \ln (\|z\|) .
$$

Rewriting this in the outer variables $y$, we obtain

$$
\left(\kappa / D_{u}\right)\left(\ln (\|y\|)+\delta^{-1}\right) .
$$

In the $\|y\|=O(1)$ region, the ansatz (3.16) (rescaled to $y$ variables), together with (3.20), results in an outer expansion for $\psi$ of the form

$$
\psi^{O}(y, \delta)=\psi_{-1}^{O}(y) \delta^{-1}+\psi_{0}^{O}(y)+O(\delta),
$$

which means that the substitution of (3.16) into (3.15) gives, at the leading order,

$$
\nabla_{y} \cdot\left(D_{u} \nabla_{y} \psi_{-1}^{O}\right)=0 \quad \text { in } Y, \quad \psi_{-1}^{O} \text { is } Y \text {-periodic, }
$$

implying that $\psi_{-1}^{O}$ is independent of $y$. At the next order in the outer expansion, we need to capture the logarithmic contribution from (3.20) (required for matching with the inner solution), and we thus conclude that

$$
\begin{aligned}
& u_{0,0} \nabla_{y} \cdot\left(D_{u} \nabla_{y} \psi_{0}^{O}\right)=\partial_{t} u_{0,0}-\nabla_{x} \cdot\left(D_{u} \nabla_{x} u_{0,0}\right)-2 \pi \kappa u_{0,0} \delta(y) \text { in } Y, \\
& \psi_{0}^{O} \quad \text { is } Y \text {-periodic. }
\end{aligned}
$$

Copyright $\odot$ by SIAM. Unauthorized reproduction of this article is prohibited. 
Due to the Fredholm alternative this problem admits a solution if and only if

$$
\partial_{t} u_{0,0}=\nabla_{x} \cdot\left(D_{u} \nabla_{x} u_{0,0}\right)-2 \pi \kappa u_{0,0} \quad \text { for } \quad x \in \Omega_{L}, t>0 .
$$

We have thus obtained an outer approximation

$$
\begin{array}{r}
u_{\varepsilon}=\left[u_{0,0}(t, x)+\cdots\right]+\varepsilon\left[U_{1,0}(t, x)+\boldsymbol{\nu}_{0}^{O}(y) \cdot \nabla_{\hat{x}} u_{0,0}(t, x)+\cdots\right] \\
+\varepsilon^{2}\left[U_{2,0}(t, x)+\delta^{-1} u_{0,0}(t, x) \psi_{-1}^{O}(y)+\cdots\right]+\cdots .
\end{array}
$$

Note as a consistency check that we could have also arrived at (3.23) more directly via the $a \rightarrow 0$ limit in (3.7) (for details, see section 4.2 in [18]). However, in general, as we have $\delta^{-1} \gg 1$, the $\varepsilon^{2} \delta^{-1}$ term could be promoted to $O(\varepsilon)$ or even $O(1)$, depending on the specified limit behavior of $\delta$ with respect to $\varepsilon \rightarrow 0$, thereby identifying the distinguished limit that we consider below.

3.2. Derivation of macroscopic equations: Distinguished limit. In the asymptotic analysis in section 3.1 we first took the limit $\varepsilon \rightarrow 0$ and then $a_{\varepsilon} \rightarrow 0$. Motivated by the $\varepsilon^{2} \delta^{-1}$ term (with $\delta^{-1}=\ln \left(1 / a_{\varepsilon}\right)$ ) from (3.24), in this section we consider the situation where $\varepsilon$ and $\ln \left(1 / a_{\varepsilon}\right)$ are dependent, and we analyze two cases, $\varepsilon \ln \left(1 / a_{\varepsilon}\right)=O(1)$ (subsection 3.2.1) and $\varepsilon^{2} \ln \left(1 / a_{\varepsilon}\right)=O(1)$ (subsection 3.2.2). Note that even though the case $\varepsilon \ln \left(1 / a_{\varepsilon}\right)=O(1)$ does not give us a distinguished limit, the $O(\varepsilon)$ balance changes, and thus this case is still worth studying. In both cases we set $K\left(a_{\varepsilon}\right)=\kappa / a_{\varepsilon}$ and use the formal asymptotic expansion

$$
u(t, x, \varepsilon)=u_{0}(t, x, \hat{x} / \varepsilon)+\varepsilon u_{1}(t, x, \hat{x} / \varepsilon)+\varepsilon^{2} u_{2}(t, x, \hat{x} / \varepsilon)+\varepsilon^{3} u_{3}(t, x, \hat{x} / \varepsilon)+\cdots
$$

to derive the macroscopic equations, where $u_{j}$ are $Y$-periodic with respect to the outer microscopic variables $y=\hat{x} / \varepsilon$. The convergence of solutions of the multiscale problems to solutions of the derived macroscopic equations will be subsequently confirmed via rigorous analysis in section 4 and numerical simulations in section 5 .

We consider a linear function $g(u)=u$ in the boundary condition (2.3); the details on derivation of the macroscopic equations for nonlinear boundary conditions are given in the supplementary material [local/web $315 \mathrm{~KB}$ ]. In the next two subsections, $\lambda$ is an $O(1)$ quantity, with a different meaning in each subsection.

3.2.1. Derivation of macroscopic equations in the case $\varepsilon \ln \left(1 / a_{\varepsilon}\right)=\lambda$. Observe first that the $\varepsilon^{2} \delta^{-1}$ term from (3.24) becomes $O(\varepsilon)$ here, and therefore we do not expect it to impact the leading order. The ansatz (3.25) yields

$$
\begin{array}{ll}
\partial_{t}\left(u_{0}+\varepsilon u_{1}+\cdots\right)=\left(\frac{1}{\varepsilon^{2}} \mathcal{A}_{0}+\frac{1}{\varepsilon} \mathcal{A}_{1}+\mathcal{A}_{2}\right)\left(u_{0}+\varepsilon u_{1}+\cdots\right) & \text { in } \Omega_{L} \times Y_{a_{\varepsilon}}, \\
D_{u}\left(\frac{1}{\varepsilon} \nabla_{y}+\nabla_{\hat{x}}\right)\left(u_{0}+\varepsilon u_{1}+\cdots\right) \cdot \hat{\mathbf{n}}=-\kappa e^{\frac{\lambda}{\varepsilon}} \varepsilon\left(u_{0}+\varepsilon u_{1}+\cdots\right) & \text { on } \Omega_{L} \times \Gamma_{a_{\varepsilon}},
\end{array}
$$

where

$\mathcal{A}_{0} v \equiv \nabla_{y} \cdot\left(D_{u} \nabla_{y} v\right), \mathcal{A}_{1} v \equiv \nabla_{y} \cdot\left(D_{u} \nabla_{\hat{x}} v\right)+\nabla_{\hat{x}} \cdot\left(D_{u} \nabla_{y} v\right), \mathcal{A}_{2} v \equiv \nabla_{x} \cdot\left(D_{u} \nabla_{x} v\right)$.

On the root surface we have

$D_{u}\left(\frac{1}{\varepsilon} \nabla_{y}+\nabla_{x}\right)\left(u_{0}+\varepsilon u_{1}+\varepsilon^{2} u_{2}+\cdots\right) \cdot \mathbf{n}=-\beta\left(u_{0}+\varepsilon u_{1}+\cdots\right) \quad$ on $\left\{x_{3}=0\right\} \times Y_{a_{\varepsilon}}$. 
As in section 3.1 we analyze the behavior of solutions for $\|z\|=O(1)$ and $\|y\|=O(1)$ successively. The scaling $z=y / a_{\varepsilon}=y e^{\lambda / \varepsilon}$ implies

$$
\begin{gathered}
\partial_{t} u_{0}+\varepsilon \partial_{t} u_{1}+\cdots=\left(\frac{e^{\frac{2 \lambda}{\varepsilon}}}{\varepsilon^{2}} \mathcal{B}_{0}+\frac{e^{\frac{\lambda}{\varepsilon}}}{\varepsilon} \mathcal{B}_{1}+\mathcal{A}_{2}\right)\left(u_{0}+\varepsilon u_{1}+\cdots\right) \text { in } \Omega_{L} \times Y_{1 / a_{\varepsilon}}, \\
D_{u}\left(\frac{e^{\frac{\lambda}{\varepsilon}}}{\varepsilon} \nabla_{z}+\nabla_{\hat{x}}\right)\left(u_{0}+\varepsilon u_{1}+\cdots\right) \cdot \hat{\mathbf{n}}=-\kappa \varepsilon e^{\frac{\lambda}{\varepsilon}}\left(u_{0}+\varepsilon u_{1}+\cdots\right) \text { on } \Omega_{L} \times \partial B_{1},
\end{gathered}
$$

where

$$
\mathcal{B}_{0} v \equiv \nabla_{z} \cdot\left(D_{u} \nabla_{z} v\right), \quad \mathcal{B}_{1} v \equiv \nabla_{z} \cdot\left(D_{u} \nabla_{\hat{x}} v\right)+\nabla_{\hat{x}} \cdot\left(D_{u} \nabla_{z} v\right)
$$

The inner approximations satisfy

$$
\begin{aligned}
& \nabla_{z} \cdot\left(D_{u} \nabla_{z} u_{j}^{I}\right)=0 \text { in } Y_{\infty}, \quad D_{u} \nabla_{z} u_{j}^{I} \cdot \hat{\mathbf{n}}=0 \quad \text { on } \partial B_{1}, j=0,1, \\
& \nabla_{z} \cdot\left(D_{u} \nabla_{z} u_{j}^{I}\right)=0 \text { in } Y_{\infty}, \quad D_{u} \nabla_{z} u_{j}^{I} \cdot \hat{\mathbf{n}}=-\kappa u_{j-2}^{I} \quad \text { on } \partial B_{1}, j=2,3,4 \text {, }
\end{aligned}
$$

which imply

$$
\begin{aligned}
& u_{0}^{I}(t, x, z)=u_{0}^{I}(t, x), \quad u_{1}^{I}(t, x, z)=u_{1}^{I}(t, x), \\
& u_{j}^{I}(t, x, z)=\frac{\kappa}{D_{u}} u_{j-2}^{I}(t, x) \ln (\|z\|)+U_{j}^{I}(t, x) \quad \text { for } \quad j=2,3, \\
& u_{4}^{I}(t, x, z)=\frac{\kappa}{D_{u}} U_{2}^{I}(t, x) \ln (\|z\|)+U_{4}^{I}(t, x) .
\end{aligned}
$$

Note that in this section we expand up to $O\left(\varepsilon^{4}\right)$, because we wish to find a twoscale approximation valid up to $O\left(\varepsilon^{2}\right)$ and compare it with full-geometry numerical simulation results in section 5 . The outer approximations satisfy

$$
\nabla_{y} \cdot\left(D_{u} \nabla_{y} u_{0}^{O}\right)=0 \quad \text { in } Y, \quad u_{0}^{O} \quad Y \text {-periodic, }
$$

so $u_{0}^{O}(t, x, y)=u_{0}^{O}(t, x)$, and therefore $u_{1}^{O}(t, x, y)=u_{1}^{O}(t, x)$ holds similarly. Since in the outer microscopic variables we have

$$
u_{2}^{I}(t, x, z)=\frac{\kappa}{D_{u}}\left[u_{0}^{I}(t, x) \ln (\|y\|)+u_{0}^{I}(t, x) \frac{\lambda}{\varepsilon}\right]+U_{2}^{I}(t, x),
$$

to match logarithmic terms in the outer and inner approximations we consider

$$
\nabla_{y} \cdot\left(D_{u} \nabla_{y} u_{2}^{O}\right)=\partial_{t} u_{0}^{O}-\nabla_{x} \cdot\left(D_{u} \nabla_{x} u_{0}^{O}\right)+2 \pi \kappa u_{0}^{I} \delta(y) \quad \text { in } Y,
$$

and $u_{2}^{O}$ is $Y$-periodic. The solvability condition for (3.32) yields

$$
\partial_{t} u_{0}^{O}=\nabla_{x} \cdot\left(D_{u} \nabla_{x} u_{0}^{O}\right)-2 \pi \kappa u_{0}^{I} \quad \text { for } x \in \Omega_{L}, t>0,
$$

and substituting this result into (3.32) gives

$$
\nabla_{y} \cdot\left(D_{u} \nabla_{y} u_{2}^{O}\right)=2 \pi \kappa(\delta(y)-1) u_{0}^{I} \quad \text { in } Y .
$$

Therefore

$$
u_{2}^{O}(t, x, y)=U_{2}^{O}(t, x)+2 \pi\left(\kappa / D_{u}\right) u_{0}^{I}(t, x) \psi(y) \quad \text { for } \quad x \in \Omega_{L}, t>0,
$$

Copyright (c) by SIAM. Unauthorized reproduction of this article is prohibited. 
where $\psi(y)$ is a solution (unique up to a constant) of

$$
\Delta_{y} \psi=\delta(y)-1 \quad \text { in } Y, \quad \psi \quad Y \text {-periodic. }
$$

For similar reasons,

$$
\begin{aligned}
\nabla_{y} \cdot\left(D_{u} \nabla_{y} u_{3}^{O}\right) & +4 \pi \kappa \nabla_{y} \psi \cdot \nabla_{\hat{x}} u_{0}^{I} \\
& =\partial_{t} u_{1}^{O}-\nabla_{x} \cdot\left(D_{u} \nabla_{x} u_{1}^{O}\right)+2 \pi \kappa u_{1}^{I} \delta(y) \quad \text { in } Y,
\end{aligned}
$$

and $u_{3}^{O}$ is $Y$-periodic. Due to the periodicity conditions imposed on $\psi$, we conclude that

$$
\partial_{t} u_{1}^{O}=\nabla_{x} \cdot\left(D_{u} \nabla_{x} u_{1}^{O}\right)-2 \pi \kappa u_{1}^{I} \quad \text { for } x \in \Omega_{L}, t>0 .
$$

At the next order, we obtain

$$
\begin{aligned}
& \nabla_{y} \cdot\left(D_{u} \nabla_{y} u_{4}^{O}\right)+\nabla_{y} \cdot\left(D_{u} \nabla_{\hat{x}} u_{3}^{O}\right)+\nabla_{\hat{x}} \cdot\left(D_{u} \nabla_{y} u_{3}^{O}\right) \\
& =\partial_{t} U_{2}^{O}-\nabla_{x} \cdot\left(D_{u} \nabla_{x} U_{2}^{O}\right)+2 \pi \frac{\kappa}{D_{u}}\left[\partial_{t} u_{0}^{I}-\nabla_{x} \cdot\left(D_{u} \nabla_{x} u_{0}^{I}\right)\right] \psi(y),
\end{aligned}
$$

and $u_{4}^{O}$ is $Y$-periodic, and to match the contribution from the inner solution we require

$$
\nabla_{y} \cdot\left(D_{u} \nabla_{y} u_{4}^{O}\right)+\nabla_{y} \cdot\left(D_{u} \nabla_{\hat{x}} u_{3}^{O}\right)+\nabla_{\hat{x}} \cdot\left(D_{u} \nabla_{y} u_{3}^{O}\right)=\partial_{t} U_{2}^{O}-\nabla_{x} \cdot\left(D_{u} \nabla_{x} U_{2}^{O}\right)
$$

$$
+2 \pi\left(\kappa / D_{u}\right)\left[\partial_{t} u_{0}^{I}-\nabla_{x} \cdot\left(D_{u} \nabla_{x} u_{0}^{I}\right)\right] \psi(y)+2 \pi \kappa U_{2}^{I} \delta(y) \quad \text { in } Y .
$$

The solvability of (3.40) implies

$$
\partial_{t} U_{2}^{O}=\nabla_{x} \cdot\left(D_{u} \nabla_{x} U_{2}^{O}\right)-2 \pi \frac{\kappa}{D_{u}}\left[\partial_{t} u_{0}^{I}-\nabla_{x} \cdot\left(D_{u} \nabla_{x} u_{0}^{I}\right)\right] f_{Y} \psi(y) d y-2 \pi \kappa U_{2}^{I}
$$

in $\Omega_{L}$ and for $t>0$. Thus we obtain the outer approximation

$$
u_{0}^{O}(t, x)+\varepsilon u_{1}^{O}(t, x)+\varepsilon^{2}\left(U_{2}^{O}(t, x)+2 \pi\left(\kappa / D_{u}\right) u_{0}^{I}(t, x) \psi(y)\right)+\cdots
$$

and the inner approximation

$$
\begin{aligned}
& u_{0}^{I}(t, x)+\varepsilon u_{1}^{I}(t, x)+\varepsilon^{2} U_{2}^{I}(t, x)+\varepsilon^{2}\left(\kappa / D_{u}\right) u_{0}^{I}(t, x) \ln (\|z\|)+\varepsilon^{3} U_{3}^{I}(t, x) \\
& +\varepsilon^{3}\left(\kappa / D_{u}\right) u_{1}^{I}(t, x) \ln (\|z\|)+\varepsilon^{4} U_{4}^{I}(t, x)+\varepsilon^{4}\left(\kappa / D_{u}\right) U_{2}^{I}(t, x) \ln (\|z\|)+\cdots .
\end{aligned}
$$

Writing the latter in terms of the outer microscopic variables $y=a_{\varepsilon} z$ gives

$$
\begin{aligned}
& u_{0}^{I}(t, x)+\varepsilon\left(u_{1}^{I}(t, x)+\lambda \frac{\kappa}{D_{u}} u_{0}^{I}(t, x)\right) \\
& +\varepsilon^{2}\left(U_{2}^{I}(t, x)+\lambda \frac{\kappa}{D_{u}} u_{1}^{I}(t, x)+\frac{\kappa}{D_{u}} u_{0}^{I}(t, x) \ln (\|y\|)\right)+\cdots .
\end{aligned}
$$

Comparing (3.42) with (3.44) at $O(1)$ and $O(\varepsilon)$ yields matching conditions

$$
\begin{aligned}
& u_{0}^{O}(t, x)=u_{0}^{I}(t, x)=u_{0}(t, x), \\
& u_{1}^{O}(t, x)=u_{1}^{I}(t, x)+\lambda\left(\kappa / D_{u}\right) u_{0}^{I}(t, x)=u_{1}^{I}(t, x)+\lambda\left(\kappa / D_{u}\right) u_{0}(t, x) .
\end{aligned}
$$

Matching the inner and outer solutions at $O\left(\varepsilon^{2}\right)$ yields

$$
U_{2}^{O}(t, x)=U_{2}^{I}(t, x)+\lambda \frac{\kappa}{D_{u}}\left[u_{1}^{O}(t, x)-\lambda \frac{\kappa}{D_{u}} u_{0}(t, x)\right],
$$

Copyright (c) by SIAM. Unauthorized reproduction of this article is prohibited. 
where we have fixed the degree of freedom in the $\psi$, satisfying (3.36), by setting

$$
\lim _{y \rightarrow 0}\{2 \pi \psi(y)-\ln (\|y\|)\}=0 .
$$

Since there are no root hairs in $\Omega \backslash \Omega_{L}$, in this part of the domain the macroscopic problem is given by the original equations. Thus, due to the continuity of concentration and fluxes on the interface $\partial \Omega_{L} \backslash \partial \Omega$ between the domain with root hairs and the domain without, we substitute (3.45) into (3.33) and obtain the macroscopic problem

$$
\begin{array}{ll}
\partial_{t} u_{0}=\nabla_{x} \cdot\left(D_{u} \nabla_{x} u_{0}\right)-2 \pi \kappa u_{0} \chi_{\Omega_{L}} & \text { in } \Omega, t>0, \\
u_{0}(0, x)=u_{\text {in }}(x) & \text { in } \Omega, \\
D_{u} \nabla_{x} u_{0} \cdot \mathbf{n}=0 & \text { on } \partial \Omega \backslash \Gamma_{R}, t>0, \\
D_{u} \nabla_{x} u_{0} \cdot \mathbf{n}=-\beta u_{0} & \text { on } \Gamma_{R}, t>0,
\end{array}
$$

where $\Gamma_{R}=\bar{\Omega} \cap\left\{x_{3}=0\right\}$, and $\chi_{\Omega_{L}}$ denotes the characteristic (or indicator) function of set $\Omega_{L}$. Notice that we obtain the same macroscopic equation as for $u_{0,0}$ in (3.23). This is because with $\varepsilon \ln \left(1 / a_{\varepsilon}\right)=O(1)$, the term $\varepsilon^{2} \delta^{-1} u_{0,0}(t, x) \psi_{-1}^{O}$ from (3.24) is promoted to $O(\varepsilon)$ but does not affect the leading order.

Substituting the second relation in (3.45) into (3.38) implies the following problem for the first-order term $u_{1}(t, x)=u_{1}^{O}(t, x)$ :

$$
\begin{array}{ll}
\partial_{t} u_{1}=\nabla_{x} \cdot\left(D_{u} \nabla_{x} u_{1}\right)-2 \pi \kappa\left\{u_{1}-\lambda\left(\kappa / D_{u}\right) u_{0}\right\} & \text { in } \Omega_{L}, t>0, \\
u_{1}(0, x)=0 & \text { in } \Omega_{L}, \\
D_{u} \nabla_{x} u_{1} \cdot \mathbf{n}=0 & \text { on } \partial \Omega_{L} \backslash \Gamma_{R}, t>0, \\
D_{u} \nabla_{x} u_{1} \cdot \mathbf{n}=-\beta u_{1} & \text { on } \Gamma_{R}, t>0 .
\end{array}
$$

Finally, we substitute (3.46) into (3.41) and obtain

$$
\begin{aligned}
& \partial_{t} U_{2}^{O}=\nabla_{x} \cdot\left(D_{u} \nabla_{x} U_{2}^{O}\right)+4 \pi^{2} \frac{\kappa^{2}}{D_{u}} u_{0} f_{Y} \psi(y) d y \\
& -2 \pi \kappa\left(U_{2}^{O}-\lambda \frac{\kappa}{D_{u}}\left[u_{1}(t, x)-\lambda \frac{\kappa}{D_{u}} u_{0}(t, x)\right]\right) \quad \text { in } \Omega_{L}, t>0, \\
& U_{2}^{O}(0, x)=-2 \pi\left(\kappa / D_{u}\right) u_{\text {in }}(x) f_{Y} \psi(y) d y \quad \text { in } \Omega_{L}, \\
& D_{u} \nabla_{x} U_{2}^{O} \cdot \mathbf{n}=-2 \pi \kappa \nabla_{x} u_{0} \cdot \mathbf{n} f_{Y} \psi(y) d y \quad \text { on } \partial \Omega_{L} \backslash \partial \Omega, \\
& D_{u} \nabla_{x} U_{2}^{O} \cdot \mathbf{n}=-\beta U_{2}^{O} \quad \text { on } \Gamma_{R}, \\
& D_{u} \nabla_{x} U_{2}^{O} \cdot \mathbf{n}=0 \quad \text { on }\left(\partial \Omega_{L} \cap \partial \Omega\right) \backslash \Gamma_{R} \text {. }
\end{aligned}
$$

Then

$$
u_{2}(t, x, y)=U_{2}^{O}(t, x)+2 \pi\left(\kappa / D_{u}\right) u_{0}(t, x) \psi(y),
$$

where $\psi$ is the solution of the "unit cell" problem (3.36) satisfying (3.47).

For the nonlinear boundary condition (2.3) on the surfaces of root hairs, together with the scaling assumption (2.5), we follow the same calculations as above and obtain

$$
\begin{array}{ll}
\partial_{t} u_{0}=\nabla_{x} \cdot\left(D_{u} \nabla_{x} u_{0}\right)-2 \pi \kappa g\left(u_{0}\right) \chi_{\Omega_{L}} & \text { in } \Omega, t>0, \\
u_{0}(0, x)=u_{\text {in }}(x) & \text { in } \Omega, \\
D_{u} \nabla_{x} u_{0} \cdot \mathbf{n}=0 & \text { on } \partial \Omega \backslash \Gamma_{R}, t>0, \\
D_{u} \nabla_{x} u_{0} \cdot \mathbf{n}=-\beta u_{0} & \text { on } \Gamma_{R}, t>0 ;
\end{array}
$$

Copyright (c) by SIAM. Unauthorized reproduction of this article is prohibited. 
see the supplementary material [local/web $315 \mathrm{~KB}$ ] for the derivation. Equations for higher-order approximations can be obtained in the same way as in the case of linear boundary conditions on the hair surfaces.

3.2.2. Derivation of macroscopic equations in the case $\varepsilon^{2} \ln \left(1 / a_{\varepsilon}\right)=\lambda$. The relation $\varepsilon^{2} \ln \left(1 / a_{\varepsilon}\right)=\lambda$ is equivalent to $a_{\varepsilon}=e^{-\lambda / \varepsilon^{2}}$. The formal asymptotic expansion (3.25) used in (2.1)-(2.3) yields

$$
\partial_{t} u_{0}+\varepsilon \partial_{t} u_{1}+\cdots=\left[\frac{1}{\varepsilon^{2}} \mathcal{A}_{0}+\frac{1}{\varepsilon} \mathcal{A}_{1}+\mathcal{A}_{2}\right]\left(u_{0}+\varepsilon u_{1}+\cdots\right) \text { in } \Omega_{L} \times Y_{a_{\varepsilon}},
$$

$$
\left[\frac{1}{\varepsilon} D_{u} \nabla_{y}+D_{u} \nabla_{\hat{x}}\right]\left(u_{0}+\varepsilon u_{1}+\cdots\right) \cdot \hat{\mathbf{n}}=-\kappa e^{\frac{\lambda}{\varepsilon^{2}}} \varepsilon\left(u_{0}+\varepsilon u_{1}+\cdots\right) \text { on } \Omega_{L} \times \Gamma_{a_{\varepsilon}} .
$$

The rescaling $z=y / a_{\varepsilon}$ implies

$$
\begin{gathered}
\partial_{t}\left(u_{0}+\varepsilon u_{1}+\cdots\right)=\left[\frac{e^{2 \lambda / \varepsilon^{2}}}{\varepsilon^{2}} \mathcal{B}_{0}+\frac{e^{\lambda / \varepsilon^{2}}}{\varepsilon} \mathcal{B}_{1}+\mathcal{A}_{2}\right]\left(u_{0}+\varepsilon u_{1}+\cdots\right) \text { in } \Omega_{L} \times Y_{1 / a_{\varepsilon}}, \\
{\left[e^{\frac{\lambda}{\varepsilon^{2}}} \varepsilon^{-1} D_{u} \nabla_{z}+D_{u} \nabla_{\hat{x}}\right]\left(u_{0}+\varepsilon u_{1}+\cdots\right) \cdot \hat{\mathbf{n}}} \\
=-\varepsilon \kappa e^{\frac{\lambda}{\varepsilon^{2}}}\left(u_{0}+\varepsilon u_{1}+\cdots\right) \text { on } \Omega_{L} \times \partial B_{1} .
\end{gathered}
$$

Then for the inner approximation we again obtain (3.29). Following the same calculations as in subsection 3.2.1, we obtain the outer approximation (3.42) and the inner approximation (3.43); writing the latter in terms of the outer variables $y$ yields

$$
\begin{aligned}
& \left(u_{0}^{I}(t, x)+\lambda \frac{\kappa}{D_{u}} u_{0}^{I}(t, x)\right)+\varepsilon\left(u_{1}^{I}(t, x)+\lambda \frac{\kappa}{D_{u}} u_{1}^{I}(t, x)\right) \\
& \quad+\varepsilon^{2}\left(\frac{\kappa}{D_{u}} u_{0}^{I}(t, x) \ln (\|y\|)+U_{2}^{I}(t, x)+\lambda \frac{\kappa}{D_{u}} U_{2}^{I}(t, x)\right)+\cdots .
\end{aligned}
$$

Matching (3.42) to (3.55) at $O(1)$ gives

$$
u_{0}^{O}(t, x)=\left(1+\lambda \kappa / D_{u}\right) u_{0}^{I}(t, x) .
$$

Substituting (3.56) into (3.33) yields the following macroscopic problem for $u_{0}(t, x)=$ $u_{0}^{O}(t, x)$ :

$$
\begin{array}{ll}
\partial_{t} u_{0}=\nabla_{x} \cdot\left(D_{u} \nabla_{x} u_{0}\right)-\frac{2 \pi \kappa}{1+\lambda \kappa / D_{u}} u_{0} \chi_{\Omega_{L}} & \text { in } \Omega, t>0, \\
u_{0}(0, x)=u_{\text {in }}(x) & \text { in } \Omega, \\
D_{u} \nabla_{x} u_{0} \cdot \mathbf{n}=-\beta u_{0} & \text { on } \Gamma_{R}, t>0, \\
D_{u} \nabla_{x} u_{0} \cdot \mathbf{n}=0 & \text { on } \partial \Omega \backslash \Gamma_{R}, t>0 .
\end{array}
$$

Notice that (3.57) differs from the macroscopic equation in (3.23), because the term $\varepsilon^{2} \delta^{-1} u_{0,0}(t, x) \psi_{-1}^{O}$ from (3.24) becomes $O(1)$ with the present scaling; for $\lambda=0$ we recover (3.23), as expected.

Comparing (3.42) with (3.55) at $O(\varepsilon)$ gives

$$
u_{1}^{O}(t, x)=\left(1+\lambda \kappa / D_{u}\right) u_{1}^{I}(t, x) .
$$

Substituting (3.58) into (3.38) implies that $u_{1}(t, x)=u_{1}^{O}(t, x)$ satisfies

$$
\begin{array}{ll}
\partial_{t} u_{1}=\nabla_{x} \cdot\left(D_{u} \nabla_{x} u_{1}\right)-\frac{2 \pi \kappa}{1+\lambda \kappa / D_{u}} u_{1} & \text { in } \Omega_{L}, t>0, \\
u_{1}(0, x)=0 & \text { in } \Omega_{L}, \\
D_{u} \nabla_{x} u_{1} \cdot \mathbf{n}=-\beta u_{1} & \text { on } \Gamma_{R}, t>0, \\
D_{u} \nabla_{x} u_{1} \cdot \mathbf{n}=0 & \text { on } \partial \Omega_{L} \backslash \Gamma_{R}, t>0,
\end{array}
$$

Copyright (c) by SIAM. Unauthorized reproduction of this article is prohibited. 
and we see that $u_{1}(t, x)=0$ (for all $t>0$ and $x \in \Omega_{L}$ ) solves this problem. Similarly,

$$
U_{2}^{O}(t, x)=\left(1+\lambda \kappa / D_{u}\right) U_{2}^{I}(t, x),
$$

together with condition (3.47) on function $\psi$. Using (3.60) in (3.41) yields

$$
\begin{array}{rrr}
\partial_{t} U_{2}^{O}= & \nabla_{x} \cdot\left(D_{u} \nabla_{x} U_{2}^{O}\right)+\frac{\kappa}{D_{u}} \frac{4 \pi^{2} \kappa u_{0}}{\left(1+\lambda\left(\kappa / D_{u}\right)\right)^{2}} f_{Y} \psi(y) d y-\frac{2 \pi \kappa}{1+\lambda\left(\kappa / D_{u}\right)} U_{2}^{O} & \text { in } \Omega_{L}, \\
& U_{2}^{O}(0, x)=-\frac{2 \pi\left(\kappa / D_{u}\right)}{1+\lambda\left(\kappa / D_{u}\right)} u_{i n}(x) f_{Y} \psi(y) d y & \text { in } \Omega_{L}, \\
(3.61) & D_{u} \nabla_{x} U_{2}^{O} \cdot \mathbf{n}=-\frac{2 \pi \kappa}{1+\lambda\left(\kappa / D_{u}\right)} \nabla_{x} u_{0} \cdot \mathbf{n} f_{Y} \psi(y) d y & \text { on } \partial \Omega_{L} \backslash \partial \Omega, \\
& D_{u} \nabla_{x} U_{2}^{O} \cdot \mathbf{n}=-\beta U_{2}^{O} \text { on } \Gamma_{R}, \quad D_{u} \nabla_{x} U_{2}^{O} \cdot \mathbf{n}=0 \quad \text { on }\left(\partial \Omega_{L} \cap \partial \Omega\right) \backslash \Gamma_{R}
\end{array}
$$

for $t>0$. Hence for $u_{2}(t, x, y)=u_{2}^{O}(t, x, y)$ we obtain

$$
u_{2}(t, x, y)=U_{2}^{O}(t, x)+\frac{2 \pi \kappa / D_{u}}{1+\lambda \kappa / D_{u}} u_{0}(t, x) \psi(y),
$$

where $\psi$ is the solution of "unit cell" problem (3.36) satisfying (3.47).

For the nonlinear boundary condition (2.3) (with the scaling assumption (2.5)), using the Taylor expansion of $g\left(u_{\varepsilon}\right)$ and following the same procedure as above gives

$$
\begin{array}{ll}
\partial_{t} u_{0}=\nabla_{x} \cdot\left(D_{u} \nabla_{x} u_{0}\right)-2 \pi \kappa g\left(h\left(u_{0}\right)\right) \chi_{\Omega_{L}} & \text { in } \Omega, t>0, \\
D_{u} \nabla_{x} u_{0} \cdot \mathbf{n}=-\beta u_{0} & \text { on } \Gamma_{R}, t>0 \\
D_{u} \nabla_{x} u_{0} \cdot \mathbf{n}=0 & \text { on } \partial \Omega \backslash \Gamma_{R}, t>0, \\
u_{0}(0, x)=u_{\text {in }}(x) & \text { in } \Omega,
\end{array}
$$

where $h=h\left(u_{0}\right)$ is the solution of $u_{0}=h+\lambda\left(\kappa / D_{u}\right) g(h)$; see the supplementary material [local/web 315KB] for the derivation. A similar result for an elliptic problem is obtained in $[14,15,16]$. Note that by choosing $g(u)=u$ we recover the effective equation from (3.57).

Assuming boundary condition (2.4), we obtain the effective equation

$$
\partial_{t} u_{0}=\nabla_{x} \cdot\left(D_{u} \nabla_{x} u_{0}\right)-2 \pi \kappa \frac{\left[\sqrt{\left(u_{0}-\tilde{\kappa}-1\right)^{2}+4 u_{0}}+u_{0}-\tilde{\kappa}-1\right]}{2+\left[\sqrt{\left(u_{0}-\tilde{\kappa}-1\right)^{2}+4 u_{0}}+u_{0}-\tilde{\kappa}-1\right]} \chi_{\Omega_{L}}
$$

for $x \in \Omega, t>0$, and $\tilde{\kappa}=\lambda \kappa / D_{u}$ (see the supplementary material [local/web 315KB]) for the derivation).

4. Rigorous derivation of macroscopic equations. In this section we give a rigorous derivation of the macroscopic equations for $(2.1)-(2.3),(2.6),(2.7)$. To prove the convergence of solutions of multiscale problem to the solution of the corresponding macroscopic equations, we first derive a priori estimates for $u_{\varepsilon}$, uniform in $\varepsilon$. Due to the nonstandard scale relation between the size and the period of the microscopic structure considered here, i.e., $a_{\varepsilon}=r_{\varepsilon} / \varepsilon \ll 1$, we need to derive modified trace estimates and extension results, taking into account the difference in the scales between $\varepsilon$ and $r_{\varepsilon}$. In the derivation of the trace estimates and extension results, we follow similar ideas as in [9], with small modifications due to the cylindrical microstructure of $\Omega^{\varepsilon}$. 
We define the following domains for some $0<\rho<1 / 2$ :

$$
\begin{aligned}
& \Omega_{0}^{\varepsilon}=\bigcup_{\xi \in \Xi^{\varepsilon}} \varepsilon\left(\bar{B}_{\rho}+\xi\right) \times(0, L), \quad \widetilde{\Omega}^{\varepsilon}=\Omega \backslash \bar{\Omega}_{0}^{\varepsilon}, \quad \widetilde{\Omega}_{L}^{\varepsilon}=\Omega_{L} \backslash \Omega_{0}^{\varepsilon}, \quad \Omega_{L}^{\varepsilon}=\Omega^{\varepsilon} \cap \Omega_{L}, \\
& \Gamma_{0}^{\varepsilon}=\bigcup_{\xi \in \Xi^{\varepsilon}} \varepsilon\left(\partial B_{\rho}+\xi\right) \times(0, L), \quad \Lambda_{0}^{\varepsilon}=\bigcup_{\xi \in \Xi^{\varepsilon}} \varepsilon\left(\partial B_{\rho}+\xi\right) .
\end{aligned}
$$

Lemma 4.1. For $v \in W^{1, p}\left(\Omega^{\varepsilon}\right)$, with $1 \leq p<\infty$, we have the following trace inequality:

$$
\frac{\varepsilon^{2}}{r_{\varepsilon}}\|v\|_{L^{p}\left(\Gamma^{\varepsilon}\right)}^{p} \leq \mu\left[\|v\|_{L^{p}\left(\widetilde{\Omega}^{\varepsilon}\right)}^{p}+\varepsilon^{p}\|\nabla v\|_{L^{p}\left(\widetilde{\Omega}^{\varepsilon}\right)}^{p}\right], \quad \mu \text {-independent of } \varepsilon, r_{\varepsilon} .
$$

Proof. For $v \in W^{1, p}\left(Y_{*} \times(0, L)\right)$ using a trace inequality [12] in $Y_{*}=Y \backslash \bar{B}_{\rho}$ (and an approximation of $v$ by smooth functions) yields

$$
\int_{\partial B_{\rho}}|v|^{p} d \gamma_{\hat{y}} \leq \mu_{1} \int_{Y_{*}}\left(|v|^{p}+\left|\nabla_{\hat{y}} v\right|^{p}\right) d \hat{y}
$$

with $\hat{y}=\left(y_{1}, y_{2}\right)$ and for a.a. $y_{3} \in(0, L)$. Scaling by $r_{\varepsilon} / \rho$ in the boundary integral and by $\varepsilon$ in the volume integral in (4.2), we obtain

$$
\frac{\rho}{r_{\varepsilon}} \int_{\partial B_{r_{\varepsilon}}}|v|^{p} d \hat{\gamma}^{\varepsilon} \leq \mu_{1} \frac{1}{\varepsilon^{2}} \int_{\varepsilon Y_{*}}\left(|v|^{p}+\varepsilon^{p}\left|\nabla_{\hat{x}} v\right|^{p}\right) d \hat{x}
$$

for $x_{3} \in(0, L)$, where $\hat{x}=\left(x_{1}, x_{2}\right), x_{1}=\varepsilon y_{1}, x_{2}=\varepsilon y_{2}$, and $x_{3}=y_{3}$. Adopting the changes of variables $x_{j} \rightarrow x_{j}+\varepsilon \xi$ in the integral over $\varepsilon Y_{*}$ and $z_{j} \rightarrow z_{j}+\varepsilon \xi$ in the boundary integral, with $j=1,2$, and multiplying by $\varepsilon^{2}$, implies

$$
\frac{\varepsilon^{2}}{r_{\varepsilon}} \int_{\partial B_{r_{\varepsilon}+\varepsilon \xi}}|v|^{p} d \hat{\gamma}^{\varepsilon} \leq \mu_{2} \int_{\varepsilon Y_{*}+\varepsilon \xi}\left(|v|^{p}+\varepsilon^{p}\left|\nabla_{\hat{x}} v\right|^{p}\right) d \hat{x} .
$$

Integrating the last inequality with respect to $x_{3}$ over $(0, L)$ and summing up over $\xi \in \Xi^{\varepsilon}$ imply the estimate (4.1).

Lemma 4.2 (extension). For $v \in H^{1}\left(\Omega^{\varepsilon}\right)$ there exists an extension $P_{\varepsilon} v \in H^{1}(\Omega)$ such that

$$
\left\|P_{\varepsilon} v\right\|_{L^{2}(\Omega)} \leq \mu\|v\|_{L^{2}\left(\Omega^{\varepsilon}\right)}, \quad\left\|\nabla P_{\varepsilon} v\right\|_{L^{2}(\Omega)} \leq \mu\|\nabla v\|_{L^{2}\left(\Omega^{\varepsilon}\right)},
$$

with a constant $\mu$ independent of $\varepsilon$.

Proof. Consider $\tilde{S}=B_{2 \rho}, S=\tilde{S} \backslash \bar{B}_{\rho}, \tilde{S}_{L}=\tilde{S} \times(0, L)$, and $S_{L}=S \times(0, L)$. By a standard extension result for $v \in H^{1}(S \times(0, L))$ there exists $\hat{v} \in H^{1}(\tilde{S} \times(0, L))$ such that

$$
\begin{aligned}
& \|\hat{v}\|_{L^{2}(\tilde{S} \times(0, L))} \leq \mu_{1}\|v\|_{L^{2}(S \times(0, L))}, \quad\|\nabla \hat{v}\|_{L^{2}(\tilde{S} \times(0, L))} \leq \mu_{1}\|\nabla v\|_{L^{2}(S \times(0, L))}, \\
& \left\|\nabla_{\hat{x}} \hat{v}\left(\cdot, x_{3}\right)\right\|_{L^{2}(\tilde{S})} \leq \mu_{1}\left\|\nabla_{\hat{x}} v\left(\cdot, x_{3}\right)\right\|_{L^{2}(S)} \quad \text { for } x_{3} \in(0, L) \text { and } \hat{x}=\left(x_{1}, x_{2}\right) ;
\end{aligned}
$$

see, e.g., [7]. Then for $v \in H^{1}\left(Y_{*}^{\varepsilon} \times(0, L)\right)$, where $Y_{*}^{\varepsilon}=\varepsilon Y \backslash \bar{B}_{r_{\varepsilon}}$, consider an extension $P_{\varepsilon}: H^{1}\left(Y_{*}^{\varepsilon} \times(0, L)\right) \rightarrow H^{1}(\varepsilon Y \times(0, L))$ such that $P_{\varepsilon} v=v$ in $Y_{*}^{\varepsilon} \times(0, L)$ and $P_{\varepsilon} v(x)=\hat{v}\left(\rho \hat{x} / r_{\varepsilon}, x_{3}\right)$ in $B_{r_{\varepsilon}} \times(0, L)$. The estimates (4.4) then give

$$
\begin{aligned}
& \int_{B_{r_{\varepsilon}} \times(0, L)}\left\|P_{\varepsilon} v\right\|^{2} d x=\frac{r_{\varepsilon}^{2}}{\rho^{2}} \int_{B_{\rho} \times(0, L)}\left\|P_{\varepsilon} v\right\|^{2} d y \leq \frac{r_{\varepsilon}^{2}}{\rho^{2}} \int_{\tilde{S}_{L}}\left\|P_{\varepsilon} v\right\|^{2} d y \\
& \leq \mu_{1} \frac{r_{\varepsilon}^{2}}{\rho^{2}} \int_{S_{L}}\left\|P_{\varepsilon} v\right\|^{2} d y \leq \mu_{1} \int_{\frac{r_{\varepsilon}}{\rho} S \times(0, L)}\left\|P_{\varepsilon} v\right\|^{2} d x \leq \mu_{1} \int_{Y_{*}^{\varepsilon} \times(0, L)}\left\|P_{\varepsilon} v\right\|^{2} d x
\end{aligned}
$$

Copyright $@$ ㅇ by SIAM. Unauthorized reproduction of this article is prohibited. 
and

$$
\begin{aligned}
& \int_{B_{r_{\varepsilon}} \times(0, L)}\left\|\nabla_{\hat{x}} P_{\varepsilon} v\right\|^{2} d x=r_{\varepsilon}^{2} r_{\varepsilon}^{-2} \int_{B_{\rho} \times(0, L)}\left\|\nabla_{\hat{y}} P_{\varepsilon} v\right\|^{2} d y \leq \int_{\tilde{S}_{L}}\left\|\nabla_{\hat{y}} P_{\varepsilon} v\right\|^{2} d y \\
& \leq \mu_{1} \int_{S_{L}}\left\|\nabla_{\hat{y}} P_{\varepsilon} v\right\|^{2} d y \leq \mu_{1} \int_{\frac{r_{\varepsilon}}{\rho} S \times(0, L)}\left\|\nabla_{\hat{x}} P_{\varepsilon} v\right\|^{2} d x \leq \mu_{1} \int_{Y_{*}^{\varepsilon} \times(0, L)}\left\|\nabla_{\hat{x}} P_{\varepsilon} v\right\|^{2} d x,
\end{aligned}
$$

where the constant $\mu_{1}$ is independent of $r_{\varepsilon}$ and $\varepsilon$, and $x_{j}=\left(r_{\varepsilon} / \rho\right) y_{j}$ for $j=1,2$, $x_{3}=y_{3}$. For the derivative with respect to $x_{3}$ we have

$$
\begin{aligned}
& \int_{B_{r_{\varepsilon}} \times(0, L)}\left\|\partial_{x_{3}} P_{\varepsilon} v\right\|^{2} d x=\frac{r_{\varepsilon}^{2}}{\rho^{2}} \int_{B_{\rho} \times(0, L)}\left\|\partial_{y_{3}} P_{\varepsilon} v\right\|^{2} d y \leq \frac{r_{\varepsilon}^{2}}{\rho^{2}} \int_{\tilde{S}_{L}}\left\|\partial_{y_{3}} P_{\varepsilon} v\right\|^{2} d y \\
& \leq \mu_{1} \frac{r_{\varepsilon}^{2}}{\rho^{2}} \int_{S_{L}}\left\|\nabla_{y} P_{\varepsilon} v\right\|^{2} d y \leq \mu_{1} \int_{\frac{r_{\varepsilon}}{\rho} S \times(0, L)}\left\|\nabla_{x} P_{\varepsilon} v\right\|^{2} d x \leq \mu_{1} \int_{Y_{*}^{\varepsilon} \times(0, L)}\left\|\nabla_{x} P_{\varepsilon} v\right\|^{2} d x .
\end{aligned}
$$

Combining the estimates above with the fact that $P_{\varepsilon} v=v$ in $Y_{*}^{\varepsilon} \times(0, L)$ yields

$$
\left\|P_{\varepsilon} v\right\|_{L^{2}(\varepsilon Y \times(0, L))} \leq \mu\|v\|_{L^{2}\left(Y_{*}^{\varepsilon} \times(0, L)\right)}, \quad\left\|\nabla P_{\varepsilon} v\right\|_{L^{2}(\varepsilon Y \times(0, L))} \leq \mu\|\nabla v\|_{L^{2}\left(Y_{*}^{\varepsilon} \times(0, L)\right)} .
$$

Considering the last inequalities for $Y_{*}^{\varepsilon}+\varepsilon \xi$ and summing up over $\xi \in \Xi^{\varepsilon}$ imply the extension and estimates stated in the lemma.

LEMma 4.3. Assume $g$ is continuously differentiable on $[-\tilde{\varsigma}, \infty)$ for some $\tilde{\varsigma}>0$, and $g(\eta)=g_{1}(\eta)+g_{2}(\eta)$, where $g_{1}(\eta) \geq 0$ for $\eta \geq 0$, with $g_{1}(0)=0$, and $g_{2}$ is sublinear, with $g_{2}(0) \leq 0$. Additionally assume that initial condition $u_{\mathrm{in}} \in H^{1}(\Omega)$, with $0 \leq u_{\text {in }} \leq u_{\max }, K\left(a_{\varepsilon}\right)=\kappa / a_{\varepsilon}$, with $\kappa>0$, and $\beta \geq 0$. Then solutions $u_{\varepsilon}$ of (2.1)-(2.3), (2.6), (2.7) satisfy the a priori estimates

$$
\begin{array}{r}
\left\|u_{\varepsilon}\right\|_{L^{\infty}\left(0, T ; L^{2}\left(\Omega^{\varepsilon}\right)\right)}^{2}+\left\|\nabla u_{\varepsilon}\right\|_{L^{2}\left((0, T) \times \Omega^{\varepsilon}\right)}^{2}+\beta\left\|u_{\varepsilon}\right\|_{L^{2}\left((0, T) \times \Gamma_{R}^{\varepsilon}\right)}^{2} \\
+\frac{\varepsilon^{2}}{r_{\varepsilon}} \int_{\Gamma_{T}^{\varepsilon}} g_{1}\left(u_{\varepsilon}\right) u_{\varepsilon} d \gamma^{\varepsilon} d t+\left\|\partial_{t} u_{\varepsilon}\right\|_{L^{2}\left((0, T) \times \Omega^{\varepsilon}\right)}^{2} \leq \mu, \\
\left\|\left(u_{\varepsilon}-M e^{m t}\right)^{+}\right\|_{L^{2}\left((0, T) \times \Omega^{\varepsilon}\right)}^{2} \leq \mu \varepsilon,
\end{array}
$$

where constants $M, m, \mu>0$ are independent of $\varepsilon$ and of $r_{\varepsilon}=\varepsilon a_{\varepsilon}$.

Proof. Using assumptions on $g$ and initial data and employing the theorem on positive invariant sets [31, Theorem 2], we obtain $u_{\varepsilon} \geq 0$ in $\Omega_{T}^{\varepsilon}$. Taking $u_{\varepsilon}$ as a test function in (2.8) and using the nonnegativity of $u_{\varepsilon}$ and assumptions on $g\left(u_{\varepsilon}\right)$ ensure

$$
\begin{aligned}
& \left\|u_{\varepsilon}(s)\right\|_{L^{2}\left(\Omega^{\varepsilon}\right)}^{2}+2 D_{u}\left\|\nabla u_{\varepsilon}\right\|_{L^{2}\left((0, s) \times \Omega^{\varepsilon}\right)}^{2}+2 \beta\left\|u_{\varepsilon}\right\|_{L^{2}\left((0, s) \times \Gamma_{R}^{\varepsilon}\right)}^{2} \\
& \quad+2 \frac{\kappa \varepsilon^{2}}{r_{\varepsilon}} \int_{\Gamma_{s}^{\varepsilon}} g_{1}\left(u_{\varepsilon}\right) u_{\varepsilon} d \gamma^{\varepsilon} d t \leq \mu_{1} \frac{\varepsilon^{2}}{r_{\varepsilon}}\left\|u_{\varepsilon}\right\|_{L^{2}\left((0, s) \times \Gamma^{\varepsilon}\right)}^{2}+\mu_{2}+\left\|u_{\varepsilon}(0)\right\|_{L^{2}\left(\Omega^{\varepsilon}\right)}^{2}
\end{aligned}
$$

for $s \in(0, T]$. Notice that if $g(\eta) \geq 0$ for $\eta \geq 0$, i.e., $g_{2} \equiv 0$, we have $\mu_{1}=\mu_{2}=$ 0 . Then using (4.1) with $p=2$ and $\|v\|_{L^{2}\left(\widetilde{\Omega}^{\varepsilon}\right)}^{2} \leq\|v\|_{L^{2}\left(\Omega^{\varepsilon}\right)}^{2}$, applying Gronwall's inequality, and taking supremum over $s \in(0, T]$ yield the first four estimates in (4.5).

Taking $\left(u_{\varepsilon}-M e^{m t}\right)^{+}$, with $M>u_{\max }$ and some $m>0$, as a test function in (2.8), and using assumptions on $g$ and inequality (4.1), with $p=2$ and $p=1$, yield

$$
\begin{aligned}
& \left\|\left(u_{\varepsilon}(s)-M e^{m s}\right)^{+}\right\|_{L^{2}\left(\Omega^{\varepsilon}\right)}^{2}+2 D_{u}\left\|\nabla\left(u_{\varepsilon}-M e^{m t}\right)^{+}\right\|_{L^{2}\left(\Omega_{s}^{\varepsilon}\right)}^{2} \\
& \quad+2 m\left\|M e^{m t}\left(u_{\varepsilon}-M e^{m t}\right)^{+}\right\|_{L^{1}\left(\Omega_{s}^{\varepsilon}\right)} \leq \mu_{1}\left\|\left(1+M e^{m t}\right)\left(u_{\varepsilon}-M e^{m t}\right)^{+}\right\|_{L^{1}\left(\Omega_{s}^{\varepsilon}\right)} \\
& \quad+\mu_{2}\left\|\left(u_{\varepsilon}-M e^{m t}\right)^{+}\right\|_{L^{2}\left(\Omega_{s}^{\varepsilon}\right)}^{2}+\varepsilon\left(1+M e^{m s}\right)\left(\mu_{3}\left\|\nabla\left(u_{\varepsilon}-M e^{m t}\right)^{+}\right\|_{L^{2}\left(\Omega_{s}^{\varepsilon}\right)}^{2}+\mu_{4}\right) .
\end{aligned}
$$

Copyright (c) by SIAM. Unauthorized reproduction of this article is prohibited. 
Choosing $m$ such that $\mu_{1}(1+M) \leq 2 m M$ and $\varepsilon$ such that $\varepsilon \mu_{3}\left(1+M e^{m T}\right) \leq 2 D_{u}$ and applying Gronwall's inequality imply the last estimate in (4.5).

Taking $\partial_{t} u_{\varepsilon}$ as a test function in (2.8) we obtain

$$
\begin{aligned}
& 2\left\|\partial_{t} u_{\varepsilon}\right\|_{L^{2}\left(\Omega_{s}^{\varepsilon}\right)}^{2}+D_{u}\left\|\nabla u_{\varepsilon}(s)\right\|_{L^{2}\left(\Omega^{\varepsilon}\right)}^{2}+\beta\left\|u_{\varepsilon}(s)\right\|_{L^{2}\left(\Gamma_{R}^{\varepsilon}\right)}^{2} \\
& \quad+2 \frac{\kappa \varepsilon^{2}}{r_{\varepsilon}} \int_{\Gamma^{\varepsilon}} G_{1}\left(u_{\varepsilon}(s)\right) d \gamma^{\varepsilon} \leq \mu_{1} \frac{\varepsilon^{2}}{r_{\varepsilon}}\left\|u_{\varepsilon}(s)\right\|_{L^{2}\left(\Gamma^{\varepsilon}\right)}^{2}+\mu_{2}+\mu_{3}\left\|u_{\text {in }}\right\|_{H^{1}\left(\Omega^{\varepsilon}\right)}^{2}
\end{aligned}
$$

for $s \in(0, T]$ and $G_{1}(\eta)=\int_{0}^{\eta} g_{1}(\xi) d \xi$ for $\eta \geq 0$. Here we used the facts that

$$
\begin{array}{ll}
\int_{\Gamma_{R, s}^{\varepsilon}} u_{\varepsilon} \partial_{t} u_{\varepsilon} d \gamma^{\varepsilon} d t=\frac{1}{2} \int_{\Gamma_{R}^{\varepsilon}}\left(\left|u_{\varepsilon}(s)\right|^{2}-\left|u_{\varepsilon}(0)\right|^{2}\right) d \gamma^{\varepsilon}, & \int_{\Gamma_{R}^{\varepsilon}}\left|u_{\varepsilon}(0)\right|^{2} d \gamma^{\varepsilon} \leq \mu_{1} u_{\max }^{2}, \\
\int_{\Gamma_{s}^{\varepsilon}} g\left(u_{\varepsilon}\right) \partial_{t} u_{\varepsilon} d \gamma^{\varepsilon} d t=\int_{\Gamma^{\varepsilon}}\left[G\left(u_{\varepsilon}(s)\right)-G\left(u_{\varepsilon}(0)\right)\right] d \gamma^{\varepsilon}, & \text { where } G(\eta)=\int_{0}^{\eta} g(\xi) d \xi,
\end{array}
$$

and that $g_{1}(\eta) \geq 0$ implies $G_{1}(\eta) \geq 0$ for $\eta \geq 0$, whereas the sublinearity of $g_{2}$ yields $\left|G_{2}(\eta)\right| \leq \mu_{2}\left(|\eta|^{2}+1\right)$, with $G_{2}(\eta)=\int_{0}^{\eta} g_{2}(\xi) d \xi$. Since $u_{\text {in }} \in H^{1}(\Omega)$ is bounded, we obtain that $u_{\text {in }}$ is bounded on $\Gamma^{\varepsilon}$ and $\Gamma_{R}^{\varepsilon}$, and the continuity of $g$ ensures that $G\left(u_{\text {in }}\right)$ is bounded on $\Gamma^{\varepsilon}$. Using (4.1) with $p=2$ in (4.7) implies the estimate for $\partial_{t} u_{\varepsilon}$.

First, we prove convergence of a sequence of solutions of the microscopic problem for $g(u)=u$. The case of a nonlinear function $g(u)$ will be considered in Theorem 4.5.

Theorem 4.4. Consider $K=\kappa / a_{\varepsilon}$ and $\varepsilon^{2} \ln \left(1 / a_{\varepsilon}\right)=\lambda$ for some $\lambda>0, \kappa>0$, $\beta \geq 0$, and consider initial condition $u_{\text {in }} \in H^{1}(\Omega)$, with $0 \leq u_{\text {in }} \leq u_{\max }$. Then a sequence $\left\{u_{\varepsilon}\right\}$ of solutions of (2.1)-(2.3), (2.6), (2.7) converges to a solution $u_{0} \in$ $L^{2}\left(0, T ; H^{1}(\Omega)\right)$ of the macroscopic problem (3.57). If $K=\kappa / a_{\varepsilon}$ and $\varepsilon \ln \left(1 / a_{\varepsilon}\right)=\lambda$ for $\lambda>0$, then a sequence $\left\{u_{\varepsilon}\right\}$ of solutions of (2.1)-(2.3), (2.6), (2.7) converges to a solution $u_{0} \in L^{2}\left(0, T ; H^{1}(\Omega)\right)$ of the macroscopic equations (3.48).

Proof. The a priori estimates (4.5) and extension Lemma 4.2 imply

$$
\left\|u_{\varepsilon}\right\|_{L^{2}\left(0, T ; H^{1}(\Omega)\right)}+\left\|\partial_{t} u_{\varepsilon}\right\|_{L^{2}((0, T) \times \Omega)} \leq \mu,
$$

with a constant $\mu$ independent of $\varepsilon$, where $u_{\varepsilon}$ is identified with its extension. Hence there exists a function $u_{0} \in L^{2}\left(0, T ; H^{1}(\Omega)\right)$, with $\partial_{t} u_{0} \in L^{2}((0, T) \times \Omega)$, such that

$$
\begin{aligned}
& u_{\varepsilon} \rightarrow u_{0} \text { weakly in } L^{2}\left(0, T ; H^{1}(\Omega)\right), \quad \partial_{t} u_{\varepsilon} \rightarrow \partial_{t} u_{0} \text { weakly in } L^{2}((0, T) \times \Omega), \\
& u_{\varepsilon} \rightarrow u_{0} \text { strongly in } L^{2}\left(0, T ; H^{s}(\Omega)\right) \text { for } s<1 \quad \text { (up to a subsequence), }
\end{aligned}
$$

where the strong convergence is ensured by the compactness of $H^{1}(\Omega) \subset H^{s}(\Omega)$ for $s<1$ and the Aubin-Lions lemma [22].

To pass to the limit as $\varepsilon \rightarrow 0$ in the weak formulation of (2.1)-(2.3), (2.6), (2.7) we need to construct an appropriate corrector to compensate the boundary conditions on $\Gamma^{\varepsilon}$. Define $w^{\varepsilon}$ to be the solution of

$$
\begin{array}{ll}
\nabla_{\hat{x}} \cdot\left(D_{u} \nabla_{\hat{x}} w^{\varepsilon}\right)=0 & \text { in } B_{\varepsilon \rho} \backslash \bar{B}_{r_{\varepsilon}}, \\
D_{u} \nabla_{\hat{x}} w^{\varepsilon} \cdot \hat{\mathbf{n}}=-\kappa\left(\varepsilon^{2} / r_{\varepsilon}\right) w^{\varepsilon} & \text { on } \partial B_{r_{\varepsilon}}, \quad w^{\varepsilon}=1 \quad \text { on } \partial B_{\varepsilon \rho},
\end{array}
$$

where $\hat{x}=\left(x_{1}, x_{2}\right)$, which can be solved explicitly to obtain, for $\hat{x} \in B_{\varepsilon \rho} \backslash \bar{B}_{r_{\varepsilon}}$,

$$
w^{\varepsilon}(\hat{x})=\frac{\kappa \varepsilon^{2}}{D_{u}+\kappa\left(\lambda+\varepsilon^{2} \ln (\rho)\right)} \ln \left(\sqrt{x_{1}^{2}+x_{2}^{2}}\right)+\frac{D_{u}+\kappa\left(\lambda-\varepsilon^{2} \ln (\varepsilon)\right)}{D_{u}+\kappa\left(\lambda+\varepsilon^{2} \ln (\rho)\right)} .
$$

Copyright (C) by SIAM. Unauthorized reproduction of this article is prohibited. 
We extend $w^{\varepsilon}$ in a trivial way to $\left(B_{\varepsilon \rho} \backslash \bar{B}_{r_{\varepsilon}}\right) \times(0, L)$ and denote it by $\hat{w}^{\varepsilon}(x)=w^{\varepsilon}(\hat{x})$. Then we extend $\hat{w}^{\varepsilon}(x)$ periodically with period $\varepsilon Y$ into $\Omega^{\varepsilon} \cap \Omega_{0}^{\varepsilon}$ and by 1 into $\widetilde{\Omega}^{\varepsilon}$.

Using $\phi=\hat{w}^{\varepsilon} \psi_{1}+\psi_{2}$ as a test function in (2.8), where $\psi_{1} \in C^{1}\left([0, T] ; C^{1}\left(\bar{\Omega}_{L}\right)\right)$, $\psi_{2} \in C^{1}\left([0, T] ; C^{1}\left(\overline{\Omega \backslash \Omega_{L}}\right)\right)$, with $\psi_{1}(t, \hat{x}, L)=\psi_{2}(t, \hat{x}, L)=0$, and extended by zero into $\Omega_{M-L, T}=(0, T) \times\left(\Omega \backslash \bar{\Omega}_{L}\right)$ and $\Omega_{L, T}=(0, T) \times \Omega_{L}$, respectively, yields

$$
\begin{aligned}
& \int_{\Omega_{L, T}^{\varepsilon}}\left[\partial_{t} u_{\varepsilon} \hat{w}^{\varepsilon} \psi_{1}+D_{u} \nabla u_{\varepsilon} \nabla\left(\hat{w}^{\varepsilon} \psi_{1}\right)\right] d x d t+\int_{\Gamma_{T}^{\varepsilon}} \frac{\varepsilon^{2} \kappa}{r_{\varepsilon}} u_{\varepsilon} \hat{w}^{\varepsilon} \psi_{1} d \gamma^{\varepsilon} d t \\
& +\int_{\Gamma_{R, T}^{\varepsilon}} \beta u_{\varepsilon} \hat{w}^{\varepsilon} \psi_{1} d \gamma^{\varepsilon} d t+\int_{\Omega_{M-L, T}}\left[\partial_{t} u_{\varepsilon} \psi_{2}+D_{u} \nabla u_{\varepsilon} \nabla \psi_{2}\right] d x d t=0 .
\end{aligned}
$$

Notice that the assumptions on $\psi_{1}$ and $\psi_{2}$ and the construction of $\hat{w}^{\varepsilon}$ ensure that $\phi \in L^{2}\left(0, T ; H^{1}\left(\Omega^{\varepsilon}\right)\right)$. The second term in the last equality can be rewritten as

$$
\begin{aligned}
& \int_{\Omega_{L, T}^{\varepsilon}} D_{u} \hat{w}^{\varepsilon} \nabla u_{\varepsilon} \nabla \psi_{1} d x d t+\int_{\Omega_{L, T}^{\varepsilon}} D_{u} \psi_{1} \nabla u_{\varepsilon} \nabla \hat{w}^{\varepsilon} d x d t=\int_{\Omega_{L, T}^{\varepsilon}} D_{u} \hat{w}^{\varepsilon} \nabla u_{\varepsilon} \nabla \psi_{1} d x d t \\
& +\int_{\widetilde{\Omega}_{L, T}^{\varepsilon}} D_{u} \psi_{1} \nabla u_{\varepsilon} \nabla \hat{w}^{\varepsilon} d x d t+\int_{\Gamma_{T}^{\varepsilon}} D_{u} u_{\varepsilon} \nabla \hat{w}^{\varepsilon} \cdot \mathbf{n} \psi_{1} d \gamma^{\varepsilon} d t+\int_{\Gamma_{0, T}^{\varepsilon}} D_{u} u_{\varepsilon} \nabla \hat{w}^{\varepsilon} \cdot \mathbf{n} \psi_{1} d \gamma^{\varepsilon} d t \\
& -\int_{0}^{T} \int_{\Omega_{L}^{\varepsilon} \backslash \widetilde{\Omega}_{L}^{\varepsilon}}\left[u_{\varepsilon} \nabla \cdot\left(D_{u} \nabla \hat{w}^{\varepsilon}\right) \psi_{1}+D_{u} u_{\varepsilon} \nabla \hat{w}^{\varepsilon} \nabla \psi_{1}\right] d x d t .
\end{aligned}
$$

By the definition of $\hat{w}^{\varepsilon}$, we have $\nabla \cdot\left(D_{u} \nabla \hat{w}^{\varepsilon}\right)=0$ in $\Omega_{L}^{\varepsilon} \backslash \widetilde{\Omega}_{L}^{\varepsilon}$ and $\nabla \hat{w}^{\varepsilon}=0$ in $\widetilde{\Omega}_{L}^{\varepsilon}$. The definition of $\hat{w}^{\varepsilon}$ also implies

$$
\left\|\nabla \hat{w}^{\varepsilon}\right\|_{L^{2}\left(\Omega_{L}^{\varepsilon}\right)} \leq \mu
$$

with some constant $\mu$ independent of $\varepsilon$. Since $\hat{w}^{\varepsilon}$ is bounded in $\Omega_{L}^{\varepsilon},\left|\Omega_{L} \backslash \Omega_{L}^{\varepsilon}\right| \rightarrow 0$ as $\varepsilon \rightarrow 0$, and $\hat{w}^{\varepsilon}=1$ in $\widetilde{\Omega}_{L}^{\varepsilon}$, we obtain that $\widetilde{w}^{\varepsilon} \rightarrow 1$ in $L^{2}\left(\Omega_{L}\right)$ strongly, where $\widetilde{w}^{\varepsilon}$ is the extension of $\hat{w}^{\varepsilon}$ by zero into $\Omega_{L} \backslash \Omega_{L}^{\varepsilon}$. Thus strong convergence of the extension of $u_{\varepsilon}$ in $L^{2}((0, T) \times \Omega)$ and weak convergence of $\nabla \hat{w}^{\varepsilon} \rightarrow 0$ in $L^{2}\left(\Omega_{L}\right)$, using the same notation for $\hat{w}^{\varepsilon}$ and its extension, ensure

$$
\lim _{\varepsilon \rightarrow 0} \int_{0}^{T} \int_{\Omega_{L}^{\varepsilon} \backslash \widetilde{\Omega}_{L}^{\varepsilon}} D_{u} u_{\varepsilon} \nabla \hat{w}^{\varepsilon} \nabla \psi_{1} d x d t=0 .
$$

Using $\left\|\nabla u_{\varepsilon}\right\|_{L^{2}\left(\Omega_{T}\right)} \leq C$ and $\left|\Omega \backslash \Omega^{\varepsilon}\right| \rightarrow 0, \widetilde{w}^{\varepsilon} \rightarrow 1$ in $L^{2}\left(\Omega_{L}\right)$, as $\varepsilon \rightarrow 0$, yields

$$
\begin{aligned}
& \int_{\Omega_{L, T}^{\varepsilon}}\left[\partial_{t} u_{\varepsilon} \hat{w}^{\varepsilon} \psi_{1}+D_{u} \hat{w}^{\varepsilon} \nabla u_{\varepsilon} \nabla \psi_{1}\right] d x d t \rightarrow \int_{\Omega_{L, T}}\left[\partial_{t} u_{0} \psi_{1}+D_{u} \nabla u_{0} \nabla \psi_{1}\right] d x d t, \\
& \int_{\Omega_{M-L, T}}\left[\partial_{t} u_{\varepsilon} \psi_{2}+D_{u} \nabla u_{\varepsilon} \nabla \psi_{2}\right] d x d t \rightarrow \int_{\Omega_{M-L, T}}\left[\partial_{t} u_{0} \psi_{2}+D_{u} \nabla u_{0} \nabla \psi_{2}\right] d x d t, \\
& \int_{\Gamma_{R, T}^{\varepsilon}} \beta u_{\varepsilon} \hat{w}^{\varepsilon} \psi_{1} d \gamma^{\varepsilon} d t \rightarrow \int_{\Gamma_{R, T}} \beta u_{0} \psi_{1} d \hat{x} d t \quad \text { as } \varepsilon \rightarrow 0,
\end{aligned}
$$

where the strong convergence of $u_{\varepsilon}$ in $L^{2}\left(0, T ; H^{s}(\Omega)\right)$, for $\frac{1}{2}<s<1$, ensures its strong convergence in $L^{2}\left((0, T) \times \Gamma_{R}\right)$. Computing $\nabla \hat{w}^{\varepsilon}$ yields

$$
D_{u} \nabla \hat{w}^{\varepsilon} \cdot \mathbf{n}=\frac{D_{u} \kappa \varepsilon / \rho}{D_{u}+\kappa\left(\lambda+\varepsilon^{2} \ln (\rho)\right)}=\frac{\kappa \varepsilon / \rho}{1+\left(\kappa / D_{u}\right)\left(\lambda+\varepsilon^{2} \ln (\rho)\right)} \quad \text { on } \Gamma_{0}^{\varepsilon} .
$$

Copyright (c) by SIAM. Unauthorized reproduction of this article is prohibited. 
Applying the two-scale convergence on $\Gamma_{0}^{\varepsilon}=\Lambda_{0}^{\varepsilon} \times(0, L)$, with a test function $\psi_{1} \in$ $C^{1}\left([0, T] ; C^{1}\left(\bar{\Omega}_{L}\right)\right)$, see, e.g., $[1,26]$, and using $\lim _{\varepsilon \rightarrow 0} \varepsilon\left\|u_{\varepsilon}-u_{0}\right\|_{L^{2}\left(\Gamma_{0, T}^{\varepsilon}\right)}^{2}=0$, ensured by the strong convergence of $u_{\varepsilon}$ in $L^{2}\left(0, T ; H^{s}(\Omega)\right)$ for $\frac{1}{2}<s<1$ (see, e.g., [29]), yield

$$
\begin{array}{r}
\lim _{\varepsilon \rightarrow 0} \int_{\Gamma_{0, T}^{\varepsilon}} D_{u} \nabla \hat{w}^{\varepsilon} \cdot \mathbf{n} u_{\varepsilon} \psi_{1} d \gamma^{\varepsilon} d t=\lim _{\varepsilon \rightarrow 0} \varepsilon \int_{\Gamma_{0, T}^{\varepsilon}} \frac{(\kappa / \rho)\left(u_{\varepsilon}-u_{0}\right) \psi_{1}}{1+\left(\kappa / D_{u}\right)\left(\lambda+\varepsilon^{2} \ln (\rho)\right)} d \gamma^{\varepsilon} d t \\
\quad+\lim _{\varepsilon \rightarrow 0} \varepsilon \int_{0}^{T} \int_{0}^{L} \int_{\Lambda_{0}^{\varepsilon}} \frac{(\kappa / \rho) u_{0} \psi_{1}}{1+\left(\kappa / D_{u}\right)\left(\lambda+\varepsilon^{2} \ln (\rho)\right)} d \hat{\gamma}^{\varepsilon} d x_{3} d t \\
=\int_{\Omega_{L, T}} \int_{\partial B_{\rho}} \frac{(\kappa / \rho) u_{0} \psi_{1}}{1+\lambda\left(\kappa / D_{u}\right)} d \hat{\gamma} d x d t=\int_{\Omega_{L, T}} \frac{2 \pi \kappa u_{0} \psi_{1}}{1+\lambda\left(\kappa / D_{u}\right)} d x d t .
\end{array}
$$

Notice that $u_{0}$ and $\psi_{1}$ are independent of $y \in \partial B_{\rho}$, and the $\varepsilon$-scaling in the boundary integrals in (4.11) is essential for the two-scale convergence on oscillating surfaces.

Using the trace inequality $\varepsilon\|v\|_{L^{2}\left(\Gamma_{0}^{\varepsilon}\right)}^{2} \leq \mu\|v\|_{H^{1}\left(\Omega_{L}\right)}^{2}$, see, e.g., [29], we have

$$
\begin{aligned}
& \left|\varepsilon \int_{\Gamma_{0, T}^{\varepsilon}} \frac{(\kappa / \rho)\left(u_{\varepsilon}-u_{0}\right) \psi_{1}}{1+\left(\kappa / D_{u}\right)\left(\lambda+\varepsilon^{2} \ln (\rho)\right)} d \gamma^{\varepsilon} d t\right| \leq \mu_{1} \varepsilon^{\frac{1}{2}}\left\|u_{\varepsilon}-u_{0}\right\|_{L^{2}\left(\Gamma_{0, T}^{\varepsilon}\right)}\left\|\psi_{1}\right\|_{L^{2}\left(0, T ; H^{1}\left(\Omega_{L}\right)\right)}, \\
& \quad \varepsilon\left\|_{1+\left(\kappa / D_{u}\right)\left(\lambda+\varepsilon^{2} \ln (\rho)\right)}\right\|_{L^{2}\left(\Gamma_{0, T}^{\varepsilon}\right)}^{2} \leq \mu_{2}\left\|u_{0}\right\|_{L^{2}\left(0, T ; H^{1}\left(\Omega_{L}\right)\right)}^{2} \leq \mu_{3}
\end{aligned}
$$

for $0<\varepsilon \leq \varepsilon_{0}$, such that $\lambda+\varepsilon_{0}^{2} \ln (\rho)>0$ with $0<\rho<1 / 2$.

Combining all the calculations from above and taking the limit as $\varepsilon \rightarrow 0$, we obtain the equation and boundary conditions in (3.57). Standard arguments (see, e.g., [30]) ensure that $u_{0}$ satisfies the initial condition in (3.57) and is a unique solution of (3.57). Hence the whole sequence $\left\{u_{\varepsilon}\right\}$ converges to $u_{0}$ as $\varepsilon \rightarrow 0$.

If $\varepsilon \ln \left(1 / a_{\varepsilon}\right)=\lambda$, then the solution of problem (4.9) is given by

$$
\begin{aligned}
& w^{\varepsilon}\left(x_{1}, x_{2}\right)=\frac{\kappa \varepsilon^{2}}{D_{u}+\kappa\left(\varepsilon \lambda+\varepsilon^{2} \ln (\rho)\right)} \ln \left(\sqrt{x_{1}^{2}+x_{2}^{2}}\right)+\frac{D_{u}+\kappa\left(\varepsilon \lambda-\varepsilon^{2} \ln (\varepsilon)\right)}{D_{u}+\kappa\left(\varepsilon \lambda+\varepsilon^{2} \ln (\rho)\right)}, \\
& D_{u} \nabla \hat{w}^{\varepsilon} \cdot \mathbf{n}=\varepsilon \frac{\kappa / \rho}{1+\left(\kappa / D_{u}\right)\left(\varepsilon \lambda+\varepsilon^{2} \ln (\rho)\right)} \quad \text { on } \Gamma_{0}^{\varepsilon} .
\end{aligned}
$$

In this case the boundary integral converges to

$$
\int_{0}^{T} \int_{\Gamma_{0}^{\varepsilon}} D_{u} \nabla \hat{w}^{\varepsilon} \cdot \mathbf{n} u_{\varepsilon} \psi_{1} d \gamma^{\varepsilon} d t \rightarrow \int_{0}^{T} \int_{\Omega_{L}} 2 \pi \kappa u_{0} \psi_{1} d x d t \quad \text { as } \varepsilon \rightarrow 0,
$$

and we obtain the macroscopic equation as in (3.48).

Now we consider the nonlinear condition (2.3) on the boundaries of the microstructure.

TheOREM 4.5. Consider $K=\kappa / a_{\varepsilon}$ for $\kappa>0$ and $\varepsilon^{2} \ln \left(1 / a_{\varepsilon}\right)=\lambda$ for some $\lambda>0$; let $g$ be continuously differentiable and monotone nondecreasing on $[-\tilde{\varsigma}, \infty)$ for some $\tilde{\varsigma}>0$; and let $g(\eta)=g_{1}(\eta)+g_{2}(\eta)$, where $g_{1}(\eta) \geq 0$ for $\eta \geq 0$, with $g_{1}(0)=0$, and $g_{2}$ is sublinear, with $g_{2}(0) \leq 0$, initial condition $u_{\mathrm{in}} \in H^{1}(\Omega)$ with $0 \leq u_{\mathrm{in}} \leq u_{\max }$, and $\beta \geq 0$. Then a sequence $\left\{u_{\varepsilon}\right\}$ of solutions of $(2.1)-(2.3)$, (2.6), (2.7) converges to a solution $u_{0} \in L^{2}\left(0, T ; H^{1}(\Omega)\right)$ of the macroscopic problem (3.63). If $K=\kappa / a_{\varepsilon}$ and $\varepsilon \ln \left(1 / a_{\varepsilon}\right)=\lambda$ for $\lambda>0$, then a sequence $\left\{u_{\varepsilon}\right\}$ of solutions of (2.1)-(2.3), (2.6), (2.7) converges to a solution $u_{0} \in L^{2}\left(0, T ; H^{1}(\Omega)\right)$ of the macroscopic equations (3.52).

Copyright $@$ by SIAM. Unauthorized reproduction of this article is prohibited. 
Proof. In the same way as in the proof of Theorem 4.4, using a priori estimates (4.5) and extension Lemma 4.2, we obtain the convergence results

$$
\begin{aligned}
& u_{\varepsilon} \rightarrow u_{0} \text { weakly in } L^{2}\left(0, T ; H^{1}(\Omega)\right), \partial_{t} u_{\varepsilon} \rightarrow \partial_{t} u_{0} \text { weakly in } L^{2}((0, T) \times \Omega), \\
& u_{\varepsilon} \rightarrow u_{0} \text { strongly in } L^{2}\left(0, T ; H^{s}(\Omega)\right) \text { for } s<1 \quad \text { (up to a subsequence), }
\end{aligned}
$$

where $u_{0} \in L^{2}\left(0, T ; H^{1}(\Omega)\right) \cap H^{1}\left(0, T ; L^{2}(\Omega)\right)$. Since $u_{\varepsilon} \geq 0$ for all $\varepsilon>0$, we have $u_{0} \geq 0$, whereas the last estimate in (4.5), together with the strong convergence of $u_{\varepsilon}$, implies $u_{0} \in L^{\infty}((0, T) \times \Omega)$.

As in the proof of Theorem 4.4, the main step is to construct an appropriate corrector to pass to the limit in the integral over the boundaries of the microstructure. Similarly to $[14,16]$, we define $w^{\varepsilon}$ to be the solution of

$$
\Delta w^{\varepsilon}=0 \text { in } B_{\varepsilon \rho} \backslash \bar{B}_{r_{\varepsilon}}, \quad w^{\varepsilon}=1 \text { on } \partial B_{r_{\varepsilon}}, \quad w^{\varepsilon}=0 \text { on } \partial B_{\varepsilon \rho} .
$$

Then we extend $w^{\varepsilon}$ by 1 into $B_{r_{\varepsilon}}$, in a trivial way into the $x_{3}$-direction for $x_{3} \in(0, L)$, by $w^{\varepsilon}(\hat{x})\left[1+\left(L-x_{3}\right) / \varepsilon\right]$ for $x_{3} \in[L, L+\varepsilon)$, then $\varepsilon Y$-periodically into $\Omega_{0}^{\varepsilon} \cup \Omega_{0, L+\varepsilon}^{\varepsilon}$, where $\Omega_{0, L+\varepsilon}^{\varepsilon}=\bigcup_{\xi \in \Xi^{\varepsilon}} \varepsilon\left(\bar{B}_{\rho}+\xi\right) \times[L, L+\varepsilon)$, and by 0 into $\widetilde{\Omega}_{L+\varepsilon}^{\varepsilon}=\widetilde{\Omega}^{\varepsilon} \backslash \Omega_{0, L+\varepsilon}^{\varepsilon}$. We denote this extension of $w^{\varepsilon}$ again by $w^{\varepsilon}$. Then $w^{\varepsilon}(x)=\ln (|\hat{x}| /(\varepsilon \rho))\left[\ln \left(r_{\varepsilon} /(\varepsilon \rho)\right)\right]^{-1}$ for $x \in \Omega^{\varepsilon} \cap \Omega_{0}^{\varepsilon}$ and $w^{\varepsilon}(x)=0$ for $x \in \widetilde{\Omega}_{L+\varepsilon}^{\varepsilon}$. The assumption on the relation between $\varepsilon$ and $a_{\varepsilon}=r_{\varepsilon} / \varepsilon$ implies

$$
\begin{aligned}
& \int_{\Omega_{L}^{\varepsilon} \backslash \widetilde{\Omega}^{\varepsilon}}\left|\nabla w^{\varepsilon}\right|^{2} d x=\frac{1}{\ln \left(\varepsilon \rho / r_{\varepsilon}\right)^{2}} \int_{\Omega_{L}^{\varepsilon} \backslash \widetilde{\Omega}^{\varepsilon}} \frac{1}{|\hat{x}|^{2}} d x \leq \frac{2 \pi \mu_{1} L}{\varepsilon^{2} \ln \left(\varepsilon \rho / r_{\varepsilon}\right)^{2}} \int_{r_{\varepsilon}}^{\varepsilon \rho} \frac{d r}{r} \leq \mu, \\
& \int_{\Omega_{0, L+\varepsilon}^{\varepsilon}}\left|\nabla w^{\varepsilon}\right|^{2} d x \leq \mu_{1} \varepsilon\left\|\nabla w^{\varepsilon}\right\|_{L^{2}\left(\Omega_{L}^{\varepsilon} \backslash \widetilde{\Omega}^{\varepsilon}\right)}^{2}+\frac{\mu_{2}}{\varepsilon}\left\|w^{\varepsilon}\right\|_{L^{2}\left(\Omega_{L}^{\varepsilon} \backslash \widetilde{\Omega}^{\varepsilon}\right)}^{2} \leq \mu \varepsilon
\end{aligned}
$$

for some constant $\mu>0$ independent of $\varepsilon$. This, together with arguments similar to Theorem 4.4, implies that $w^{\varepsilon} \rightarrow 0$ weakly in $H^{1}(\Omega)$ and strongly in $H^{s}(\Omega)$ for $s<1$.

To prove convergence of solutions of problem (2.1)-(2.3), (2.6), (2.7), by using the monotonicity of $g$, we rewrite its weak formulation (2.8) as variational inequality

$$
\begin{aligned}
\int_{\Omega_{T}^{\varepsilon}}\left[\partial_{t} u_{\varepsilon}\left(\phi-u_{\varepsilon}\right)+D_{u} \nabla \phi \nabla\left(\phi-u_{\varepsilon}\right)\right] d x d t & +\frac{\varepsilon^{2} \kappa}{r_{\varepsilon}} \int_{\Gamma_{T}^{\varepsilon}} g(\phi)\left(\phi-u_{\varepsilon}\right) d \gamma^{\varepsilon} d t \\
& +\int_{\Gamma_{R, T}^{\varepsilon}} \beta \phi\left(\phi-u_{\varepsilon}\right) d \gamma^{\varepsilon} d t \geq 0
\end{aligned}
$$

for any $\phi \in L^{2}\left(0, T ; H^{1}\left(\Omega^{\varepsilon}\right)\right) \cap L^{\infty}\left((0, T) \times \Omega^{\varepsilon}\right)$, with $\phi(t, x) \geq-\tilde{\varsigma}$ in $(0, T) \times \Omega^{\varepsilon}$. Notice that the last condition on $\phi$ is not needed if $g$ is monotone on $\mathbb{R}$.

Considering $\phi=\psi-\tilde{\kappa} g(h) w^{\varepsilon}$, for $\psi \in C^{1}\left([0, T] ; C^{1}(\bar{\Omega})\right)$ with $\psi(t, x) \geq-\tilde{\varsigma}$ in $[0, T] \times \bar{\Omega}$, as a test function in (4.15), where $\tilde{\kappa}=\lambda \kappa / D_{u}$ and $h$ is the solution of $h+\tilde{\kappa} g(h)=\psi$, and using the weak and strong convergences of $w^{\varepsilon}$ and of the extension of $u_{\varepsilon}$, in the corresponding spaces, together with $\left|\Omega \backslash \Omega^{\varepsilon}\right| \rightarrow 0$ as $\varepsilon \rightarrow 0$, we obtain

$$
\begin{aligned}
& \lim _{\varepsilon \rightarrow 0} \int_{\Omega_{T}^{\varepsilon}} \partial_{t} u_{\varepsilon}\left(\psi-\tilde{\kappa} g(h) w^{\varepsilon}-u_{\varepsilon}\right) d x d t=\int_{\Omega_{T}} \partial_{t} u_{0}\left(\psi-u_{0}\right) d x d t \\
& \lim _{\varepsilon \rightarrow 0} \int_{\Gamma_{R, T}^{\varepsilon}} \beta\left(\psi-\tilde{\kappa} g(h) w^{\varepsilon}\right)\left(\psi-\tilde{\kappa} g(h) w^{\varepsilon}-u_{\varepsilon}\right) d \gamma^{\varepsilon} d t=\int_{\Gamma_{R, T}} \beta \psi\left(\psi-u_{0}\right) d \hat{x} d t .
\end{aligned}
$$

Copyright (c) by SIAM. Unauthorized reproduction of this article is prohibited. 
Here and in what follows we use the same notation for $u_{\varepsilon}$ and its extension. For the second term in (4.15), the weak convergence of $\nabla u_{\varepsilon}$ and $\left|\Omega \backslash \Omega^{\varepsilon}\right| \rightarrow 0$, as $\varepsilon \rightarrow 0$, yield

$$
\begin{array}{r}
\lim _{\varepsilon \rightarrow 0} \int_{\Omega_{T}^{\varepsilon}} D_{u} \nabla\left(\psi-\tilde{\kappa} g(h) w^{\varepsilon}\right) \nabla\left(\psi-\tilde{\kappa} g(h) w^{\varepsilon}-u_{\varepsilon}\right) d x d t=\int_{\Omega_{T}} D_{u} \nabla \psi \nabla\left(\psi-u_{0}\right) d x d t \\
-\lim _{\varepsilon \rightarrow 0} \int_{\Omega_{T}^{\varepsilon}} D_{u} \tilde{\kappa}\left(\nabla g(h) w^{\varepsilon}+g(h) \nabla w^{\varepsilon}\right) \nabla\left(\psi-\tilde{\kappa} g(h) w^{\varepsilon}-u_{\varepsilon}\right) d x d t .
\end{array}
$$

For the first part of the last term the strong convergence of $w^{\varepsilon}$ and weak convergence of $\nabla w^{\varepsilon}$ and $\nabla u_{\varepsilon}$ in $L^{2}\left(\Omega_{T}\right)$ ensure

$$
\lim _{\varepsilon \rightarrow 0} \int_{\Omega_{T}^{\varepsilon}} D_{u} \tilde{\kappa} \nabla g(h) w^{\varepsilon} \nabla\left(\psi-\tilde{\kappa} g(h) w^{\varepsilon}-u_{\varepsilon}\right) d x d t=0,
$$

and the second part can be rewritten as

$$
\begin{array}{r}
\int_{\Omega_{T}^{\varepsilon}} D_{u} \tilde{\kappa}\left[\nabla w^{\varepsilon} \nabla\left(g(h)\left[\psi-\tilde{\kappa} g(h) w^{\varepsilon}-u_{\varepsilon}\right]\right)-\nabla w^{\varepsilon} \nabla g(h)\left(\psi-\tilde{\kappa} g(h) w^{\varepsilon}-u_{\varepsilon}\right)\right] d x d t \\
=I_{1}+I_{2},
\end{array}
$$

where $\lim _{\varepsilon \rightarrow 0} I_{2}=0$, due to weak convergence of $\nabla w^{\varepsilon}$ and strong convergence of $u_{\varepsilon}$ and $w^{\varepsilon}$ in $L^{2}\left(\Omega_{T}\right)$. Using that $\Delta w^{\varepsilon}=0$ in $\Omega^{\varepsilon} \cap \Omega_{0}^{\varepsilon}$ and $\nabla w^{\varepsilon}=0$ in $\Omega^{\varepsilon} \backslash\left(\Omega_{0}^{\varepsilon} \cup \Omega_{0, L+\varepsilon}^{\varepsilon}\right)$ and integrating by parts in $I_{1}$ yield

$$
I_{1}=\frac{\lambda \kappa}{\lambda+\varepsilon^{2} \ln (\rho)}\left[\frac{\varepsilon^{2}}{r_{\varepsilon}} \int_{\Gamma_{T}^{\varepsilon}} g(h)\left(\psi-\tilde{\kappa} g(h)-u_{\varepsilon}\right) d \gamma^{\varepsilon} d t-\frac{\varepsilon}{\rho} \int_{\Gamma_{0, T}^{\varepsilon}} g(h)\left(\psi-u_{\varepsilon}\right) d \gamma^{\varepsilon} d t\right]+I_{11},
$$

where, due to $\lim _{\varepsilon \rightarrow 0}\left\|\nabla w^{\varepsilon}\right\|_{L^{2}\left(\Omega_{0, L+\varepsilon}^{\varepsilon}\right)}=0$, we have

$$
I_{11}=\int_{0}^{T} \int_{\Omega_{0, L+\varepsilon}^{\varepsilon}} D_{u} \tilde{\kappa} \nabla w^{\varepsilon} \nabla\left(g(h)\left[\psi-\tilde{\kappa} g(h) w^{\varepsilon}-u_{\varepsilon}\right]\right) d x d t \rightarrow 0 \quad \text { as } \quad \varepsilon \rightarrow 0 .
$$

Similarly to the proof of Theorem 4.4, using the two-scale convergence on $\Gamma_{0}^{\varepsilon}$ (see, e.g., $[1,26]$ ) and the fact that $\lim _{\varepsilon \rightarrow 0} \varepsilon\left\|u_{\varepsilon}-u_{0}\right\|_{L^{2}\left(\Gamma_{0, T}^{\varepsilon}\right)}^{2}=0$ (see, e.g., [29]), we obtain

$$
\begin{array}{r}
\lim _{\varepsilon \rightarrow 0} \varepsilon \frac{\lambda(\kappa / \rho)}{\lambda+\varepsilon^{2} \ln (\rho)} \int_{\Gamma_{0, T}^{\varepsilon}} g(h)\left(\psi-u_{\varepsilon}\right) d \gamma^{\varepsilon} d t=\lim _{\varepsilon \rightarrow 0} \frac{\lambda(\kappa / \rho)}{\lambda+\varepsilon^{2} \ln (\rho)} \varepsilon \int_{\Gamma_{0, T}^{\varepsilon}} g(h)\left(u_{0}-u_{\varepsilon}\right) d \gamma^{\varepsilon} d t \\
\quad+\lim _{\varepsilon \rightarrow 0} \frac{\lambda(\kappa / \rho)}{\lambda+\varepsilon^{2} \ln (\rho)} \varepsilon \int_{\Gamma_{0, T}^{\varepsilon}} g(h)\left(\psi-u_{0}\right) d \gamma^{\varepsilon} d t=2 \pi \kappa \int_{\Omega_{L, T}} g(h)\left(\psi-u_{0}\right) d x d t .
\end{array}
$$

Notice that the regularity $g(h) \in C^{1}\left([0, T] ; C^{1}(\bar{\Omega})\right)$, ensured by the regularity of $g$ and $\psi$, and the trace estimate $\varepsilon\|v\|_{L^{2}\left(\Gamma_{0}^{\varepsilon}\right)}^{2} \leq \mu\|v\|_{H^{1}\left(\Omega_{L}\right)}^{2}$ (see, e.g., [29]) yield

$$
\begin{aligned}
& \left|\frac{\lambda(\kappa / \rho)}{\lambda+\varepsilon^{2} \ln (\rho)} \varepsilon \int_{\Gamma_{0, T}^{\varepsilon}} g(h)\left(u_{0}-u_{\varepsilon}\right) d \gamma^{\varepsilon} d t\right| \leq \mu_{1} \varepsilon^{\frac{1}{2}}\left\|u_{0}-u_{\varepsilon}\right\|_{L^{2}\left(\Gamma_{0, T}^{\varepsilon}\right)}\|g(h)\|_{L^{2}\left(0, T ; H^{1}(\Omega)\right)}, \\
& \varepsilon\left\|\frac{\lambda(\kappa / \rho)}{\lambda+\varepsilon^{2} \ln (\rho)}\left(\psi-u_{0}\right)\right\|_{L^{2}\left(\Gamma_{0, T}^{\varepsilon}\right)}^{2} \leq \mu_{2}\left[\left\|u_{0}\right\|_{L^{2}\left(0, T ; H^{1}(\Omega)\right)}^{2}+\|\psi\|_{L^{2}\left(0, T ; H^{1}(\Omega)\right)}^{2}\right] \leq \mu_{3}
\end{aligned}
$$

for $0<\varepsilon \leq \varepsilon_{0}$, with $\lambda+\varepsilon_{0}^{2} \ln (\rho)>0$ and $0<\rho<1 / 2$. It remains to show that

$$
\frac{\kappa \varepsilon^{2}}{r_{\varepsilon}} \int_{\Gamma_{T}^{\varepsilon}}\left(g(\psi-\tilde{\kappa} g(h))-\frac{\lambda}{\lambda+\varepsilon^{2} \ln (\rho)} g(h)\right)\left[\psi-\tilde{\kappa} g(h)-u_{\varepsilon}\right] d \gamma^{\varepsilon} d t \rightarrow 0 \text { as } \varepsilon \rightarrow 0 .
$$

Copyright $@$ ㅇ by SIAM. Unauthorized reproduction of this article is prohibited. 
Since $h$ is the solution of $h+\tilde{\kappa} g(h)=\psi$ and $g$ is monotone and continuous, we have

$$
\frac{\kappa \varepsilon^{2}}{r_{\varepsilon}} \int_{\Gamma_{T}^{\varepsilon}}[g(\psi-\tilde{\kappa} g(h))-g(h)]\left[\psi-\tilde{\kappa} g(h)-u_{\varepsilon}\right] d \gamma^{\varepsilon} d t=0 .
$$

The trace estimate (4.1) yields

$$
\begin{aligned}
& {\left[\frac{\lambda}{\lambda+\varepsilon^{2} \ln (\rho)}-1\right] \frac{\kappa \varepsilon^{2}}{r_{\varepsilon}} \int_{\Gamma_{T}^{\varepsilon}}\left|g(h) \| \psi-\tilde{\kappa} g(h)-u_{\varepsilon}\right| d \gamma^{\varepsilon} d t \leq \mu\left[\|h\|_{L^{2}\left(0, T ; H^{1}\left(\widetilde{\Omega}_{L}^{\varepsilon}\right)\right)}^{2}\right.} \\
& \left.\quad+\|\psi\|_{L^{2}\left(0, T ; H^{1}\left(\widetilde{\Omega}_{L}^{\varepsilon}\right)\right)}^{2}+\left\|u_{\varepsilon}\right\|_{L^{2}\left(0, T ; H^{1}\left(\widetilde{\Omega}_{L}^{\varepsilon}\right)\right)}^{2}+1\right]\left[\frac{\lambda}{\lambda+\varepsilon^{2} \ln (\rho)}-1\right] \rightarrow 0 \text { as } \varepsilon \rightarrow 0 .
\end{aligned}
$$

Collecting all calculations from above, taking the limit as $\varepsilon \rightarrow 0$ in (4.15), with $\phi=\psi-\tilde{\kappa} g(h) w^{\varepsilon}$, and employing a density argument, we obtain

$$
\begin{aligned}
\int_{\Omega_{T}}\left[\partial_{t} u_{0}\left(\psi-u_{0}\right)+D_{u} \nabla \psi \nabla\left(\psi-u_{0}\right)\right] d x d t & +\int_{\Omega_{L, T}} 2 \pi \kappa g(h)\left(\psi-u_{0}\right) d x d t \\
& +\int_{\Gamma_{R, T}} \beta \psi\left(\psi-u_{0}\right) d \hat{x} d t \geq 0
\end{aligned}
$$

for any $\psi \in L^{2}\left(0, T ; H^{1}(\Omega)\right) \cap L^{\infty}((0, T) \times \Omega)$. By choosing $\psi=u_{0} \pm \sigma \varphi$ for $\sigma>0$ and $\varphi \in L^{2}\left(0, T ; H^{1}(\Omega)\right) \cap L^{\infty}((0, T) \times \Omega)$, and letting $\sigma \rightarrow 0$ we obtain that $u_{0}$ is a solution of the macroscopic problem (3.63). Since $u_{0} \geq 0$, we have $\psi \geq-\tilde{\varsigma}$ for sufficiently small $\sigma$. Standard calculations ensure uniqueness of a solution of (3.63).

If $K=\kappa / a_{\varepsilon}$ and $\varepsilon \ln \left(1 / a_{\varepsilon}\right)=\lambda$, we again rewrite (2.1)-(2.3), (2.6), (2.7) as variational inequality (4.15). The convergence, as $\varepsilon \rightarrow 0$, of the first two terms and of the last integral in (4.15) follows directly from the weak convergence $u_{\varepsilon} \rightarrow u_{0}$ in $L^{2}\left(0, T ; H^{1}(\Omega)\right) \cap H^{1}\left(0, T ; L^{2}(\Omega)\right)$ and $\left|\Omega \backslash \Omega^{\varepsilon}\right| \rightarrow 0$ as $\varepsilon \rightarrow 0$. To show

$$
\lim _{\varepsilon \rightarrow 0} \frac{\varepsilon^{2} \kappa}{r_{\varepsilon}} \int_{\Gamma_{T}^{\varepsilon}} g(\phi)\left(\phi-u_{\varepsilon}\right) d \gamma^{\varepsilon} d t=2 \pi \kappa \int_{\Omega_{L, T}} g(\phi)\left(\phi-u_{0}\right) d x d t
$$

we consider the solution of the problem

$$
\nabla \cdot\left(D_{u} \nabla \tilde{w}^{\varepsilon}\right)=0 \text { in } B_{\varepsilon \rho} \backslash \bar{B}_{r_{\varepsilon}}, \quad D_{u} \nabla \tilde{w}^{\varepsilon} \cdot \nu=\frac{\varepsilon^{2} \kappa}{r_{\varepsilon}} \text { on } \partial B_{r_{\varepsilon}}, \quad \tilde{w}^{\varepsilon}=0 \text { on } \partial B_{\varepsilon \rho},
$$

given by $\tilde{w}^{\varepsilon}=\varepsilon^{2}\left(\kappa / D_{u}\right) \ln (|\hat{x}| /(\varepsilon \rho))$, extended in a trivial way to $\left(B_{\varepsilon \rho} \backslash \bar{B}_{r_{\varepsilon}}\right) \times(0, L)$ and then $\varepsilon Y$-periodically into $\Omega^{\varepsilon} \cap \Omega_{0}^{\varepsilon}$. Note that $\left|\tilde{w}^{\varepsilon}(x)\right| \leq\left(\kappa / D_{u}\right) \varepsilon^{2} \ln \left(\varepsilon \rho / r_{\varepsilon}\right) \leq \mu \varepsilon$ for all $x \in \Omega^{\varepsilon} \cap \Omega_{0}^{\varepsilon}$, and that

$$
\int_{\Omega^{\varepsilon} \cap \Omega_{0}^{\varepsilon}}\left|\nabla \tilde{w}^{\varepsilon}\right|^{2} d x \leq \mu_{1} \varepsilon^{2} \int_{r_{\varepsilon}}^{\varepsilon \rho} \frac{1}{r} d r \leq \mu \varepsilon
$$

with a constant $\mu>0$ independent of $\varepsilon$. Then

$$
\begin{array}{r}
0=-\int_{0}^{T} \int_{\Omega^{\varepsilon} \cap \Omega_{0}^{\varepsilon}} \nabla \cdot\left(D_{u} \nabla \tilde{w}^{\varepsilon}\right) g(\phi)\left(\phi-u_{\varepsilon}\right) d x d t=\int_{0}^{T} \int_{\Omega^{\varepsilon} \cap \Omega_{0}^{\varepsilon}} D_{u} \nabla \tilde{w}^{\varepsilon} \nabla\left[g(\phi)\left(\phi-u_{\varepsilon}\right)\right] d x d t \\
+\frac{\varepsilon^{2} \kappa}{r_{\varepsilon}} \int_{\Gamma_{T}^{\varepsilon}} g(\phi)\left(\phi-u_{\varepsilon}\right) d \gamma^{\varepsilon} d t-\varepsilon \frac{\kappa}{\rho} \int_{\Gamma_{0, T}^{\varepsilon}} g(\phi)\left(\phi-u_{\varepsilon}\right) d \gamma^{\varepsilon} d t .
\end{array}
$$

Copyright (c) by SIAM. Unauthorized reproduction of this article is prohibited. 
Hence taking in the last equality the limit as $\varepsilon \rightarrow 0$ and using weak convergence of $u_{\varepsilon}$ in $L^{2}\left(0, T ; H^{1}(\Omega)\right)$ and two-scale convergence on $\Gamma_{0}^{\varepsilon}$, together with the fact that $\lim _{\varepsilon \rightarrow 0}\left\|\nabla \tilde{w}^{\varepsilon}\right\|_{L^{2}\left(\Omega^{\varepsilon} \cap \Omega_{0}^{\varepsilon}\right)}=0$, imply (4.17). By choosing $\phi=u_{0} \pm \sigma \varphi$ for $\sigma>0$ and $\varphi \in L^{2}\left(0, T ; H^{1}(\Omega)\right) \cap L^{\infty}((0, T) \times \Omega)$, and letting $\sigma \rightarrow 0$ we obtain that $u_{0}$ is the solution of the macroscopic problem (3.52). Notice that in the case $\varepsilon \ln \left(1 / a_{\varepsilon}\right)=\lambda$ we can also show convergence of solutions of (2.1)-(2.3), (2.6), (2.7) directly, without rewriting it as a variational inequality and using monotonicity of $g$.

5. Numerical simulations for multiscale and macroscopic models. In this section we present numerical simulations of (2.1)-(2.3), (2.6), (2.7) and of the zero-, first-, and second-order approximations of solutions of the macroscopic problems; see (3.57), (3.59), (3.61). All simulations in this section were performed using standard finite element methods as implemented in FEniCS [23], with meshed domains generated using NETGEN [32]. Steady-state (elliptic) problems were solved directly, while for time-dependent (parabolic) problems, backwards Euler discretization in time was used, and the solution at time $t+\Delta t$ was calculated using the stationary solver with the solution at time $t$ entering the right-hand side of the weak formulation as a given forcing term (as described in [23]). Since the scale relation $\varepsilon^{2} \ln \left(1 / a_{\varepsilon}\right)=\lambda$ for small $\varepsilon$ results in a very small value for $a_{\varepsilon}$, which is numerically challenging, we consider (only) $\varepsilon=0.5$ and observe that $a_{\varepsilon}=0.01$ with such $\varepsilon$ gives $\lambda=\varepsilon^{2} \ln \left(1 / a_{\varepsilon}\right) \approx 1.15$. A continuous Galerkin finite element method of degree 1 was used, and tetrahedral meshes for the full-geometry simulations were created using in-built NETGEN generators with automatic mesh refinement close to the root hair, so that the size of any tetrahedron does not exceed 0.03, which in the case of $a_{\varepsilon}=10^{-3}$ (see below) yielded $O\left(7 \times 10^{5}\right)$ tetrahedra. For the macroscopic problems in our two-scale expansions (i.e., $u_{0}, u_{1}$, and $U_{2}$ ), we generated meshes with the maximum mesh size of 0.05 , which yielded $O(14000)$ tetrahedra for the mesh for domain $\Omega$, and $O(7000)$ for the mesh for domain $\Omega_{L}$.

We first consider the steady-state problem for (2.1), imposing a constant level of nutrient at the cut-off distance

$$
u_{\varepsilon}(t, x)=1 \quad \text { on } x_{3}=M, t>0,
$$

and a zero-flux boundary condition on $\partial \Omega^{\epsilon} \cap \partial \Omega \backslash\left\{x_{3}=M\right\}$, i.e., $\beta=0$. Then in the corresponding macroscopic problem we have

$$
u_{0}(t, x)=1 \quad \text { on } x_{3}=M, \quad D_{u} \nabla u_{0}(t, x) \cdot \mathbf{n}=0 \quad \text { on } \partial \Omega \backslash\left\{x_{3}=M\right\}, t>0 .
$$

Notice that the choice of boundary condition on $x_{3}=M$ does not affect the derivations of macroscopic equations in sections 3 and 4 . The symmetries of the full-geometry problem and the periodicity of the microstructure ensure that the solution of this problem has the same behavior in each periodicity cell $\varepsilon(Y+\xi) \times(0, M)$, for $\xi \in \mathbb{Z}^{2}$; see Figure SM1 in the supplementary material [local/web 315KB]. Hence it is sufficient to determine the solution within a single periodicity cell $\varepsilon Y \times(0, M)$.

To illustrate the differences in the behavior of the multiscale solutions and those of the corresponding macroscopic problems (3.48) and (3.57) for two different scale relations between $\varepsilon$ and $a_{\varepsilon}$, we vary $a_{\varepsilon}$ from $10^{-1}$ to $10^{-3}$; see Figure 2 . The default parameter values used throughout this section are summarized in Table 1.

For $a_{\varepsilon}=10^{-1}$ (Figure 2(b)), the steady-state solution of problem (3.48) (Figure 2(a)) gives a good averaged approximation to that of (2.1)-(2.3), (2.6), (2.7), whereas for $a_{\varepsilon}=10^{-2}$ and $a_{\varepsilon}=10^{-3}$ (Figure 2(c),(d)) the differences between the 


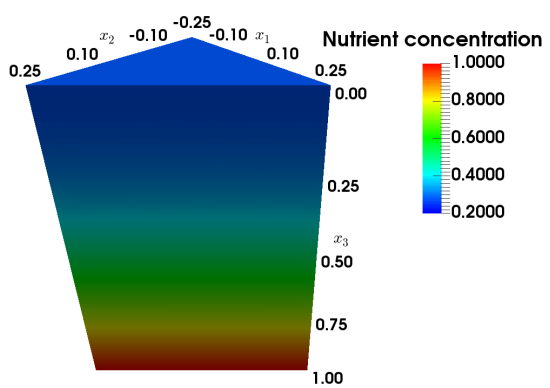

(a) $u_{0}$ for $\varepsilon \ln \left(1 / a_{\varepsilon}\right)=O(1)$

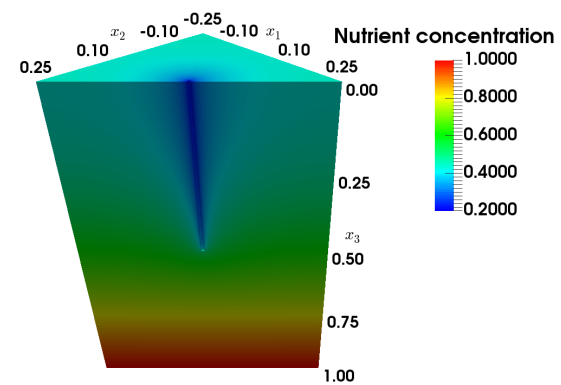

(c) $u_{\varepsilon}$ for $a_{\varepsilon}=10^{-2}\left(\varepsilon^{2} \ln \left(1 / a_{\varepsilon}\right) \approx 1.15\right)$

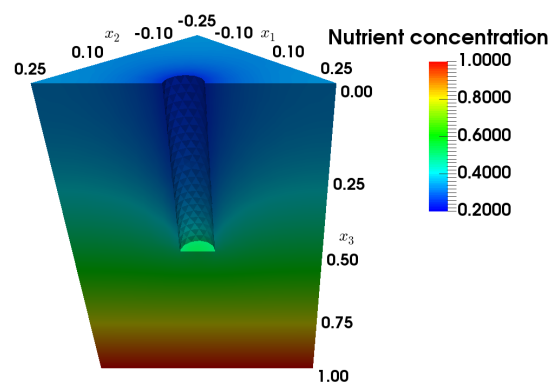

(b) $u_{\varepsilon}$ for $a_{\varepsilon}=10^{-1}\left(\varepsilon^{2} \ln \left(1 / a_{\varepsilon}\right) \approx 0.58\right)$

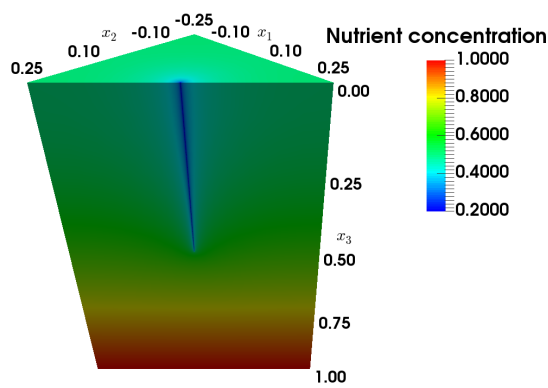

(d) $u_{\varepsilon}$ for $a_{\varepsilon}=10^{-3}\left(\varepsilon^{2} \ln \left(1 / a_{\varepsilon}\right) \approx 1.73\right)$

FiG. 2. Steady-state solutions of the macroscopic problem (3.48), (a), and of the full model (2.1)-(2.3), (2.6), (2.7), for (b) $a_{\varepsilon}=10^{-1}$, (c) $a_{\varepsilon}=10^{-2}$, and (d) $a_{\varepsilon}=10^{-3}$, with Dirichlet boundary condition (5.1), $g\left(u_{\varepsilon}\right)=u_{\varepsilon}$; all other parameters are as in Table 1 .

TABLE 1

Default dimensionless parameter values used in the numerical simulations.

\begin{tabular}{ccccccc}
\hline Parameter & $\varepsilon$ & $L$ & $M$ & $\beta$ & $D_{u}$ & $\kappa$ \\
\hline Value & 0.5 & 0.5 & 1.0 & 0.0 & 1.0 & 1.0
\end{tabular}

solution of the macroscopic problem (3.48) and those of (2.1)-(2.3), (2.6), (2.7) become more significant and, as $\varepsilon^{2} \ln \left(1 / a_{\varepsilon}\right)$ approaches 1 , the steady-state solution of the macroscopic problem (3.57) provides a better approximation to solutions of the full model, as predicted. The analysis in subsection 3.2.1 implies that for any scale relations satisfying $a_{\varepsilon} \gg e^{-1 / \varepsilon^{2}}$ as $\varepsilon \rightarrow 0$ the same macroscopic equation (3.48) pertains.

We now compare these solutions at a fixed distance from the root surface. First, we fix $x_{3}=0$ and plot the solutions along a diagonal joining the opposite corners of this plane. This way, we study behavior at the root surface, and the results for decreasing $a_{\varepsilon}$ are shown in Figure 3(a),(c),(e). Solutions of the full problem (2.1)-(2.3), (2.6), (2.7) (blue) show nutrient depletion zones close to the hair surface with increasingly sharp concentration gradients for a decreasing value of $a_{\varepsilon}$ due to the scaling of the uptake constant (2.5). Numerical simulations reveal that the steady-state solution of the macroscopic problem (3.48) underestimates, and that of the macroscopic 


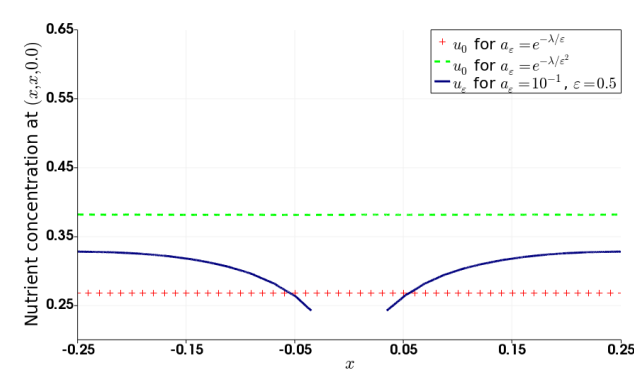

(a) $x_{3}=0.0, a_{\varepsilon}=10^{-1}$

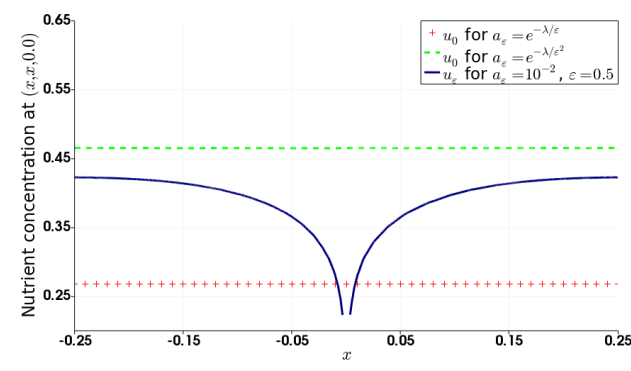

(c) $x_{3}=0.0, a_{\varepsilon}=10^{-2}$

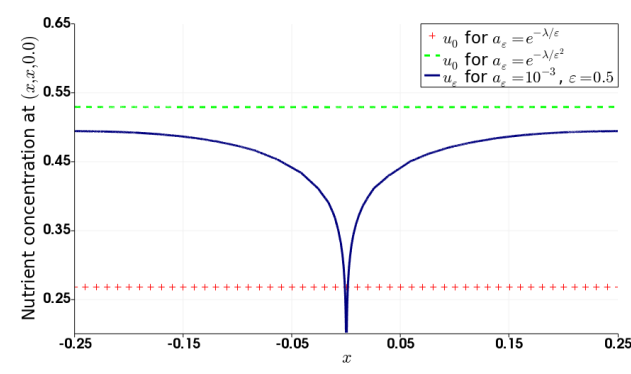

(e) $x_{3}=0.0, a_{\varepsilon}=10^{-3}$

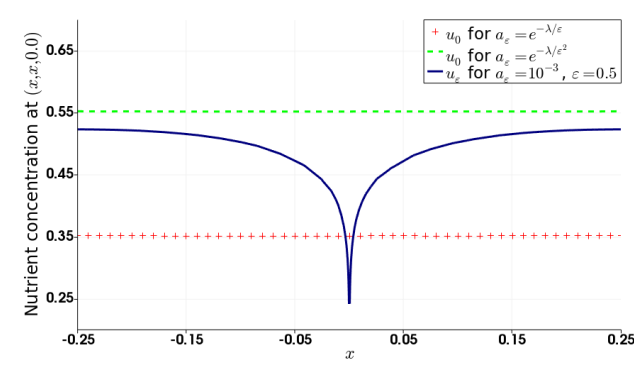

(g) $x_{3}=0.0, a_{\varepsilon}=10^{-3}, g(u)=u /(1+u)$

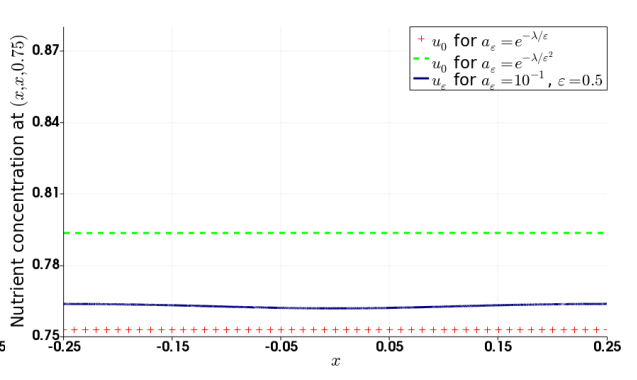

(b) $x_{3}=0.75, a_{\varepsilon}=10^{-1}$

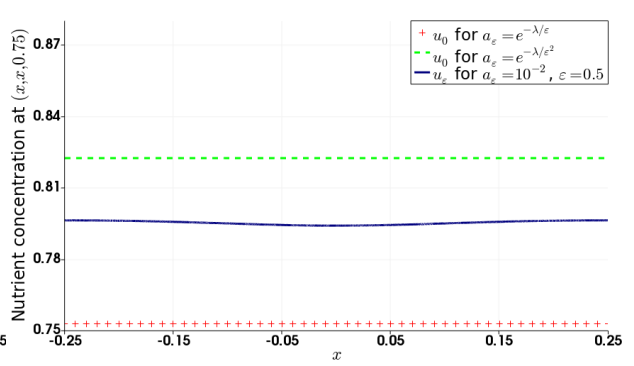

(d) $x_{3}=0.75, a_{\varepsilon}=10^{-2}$

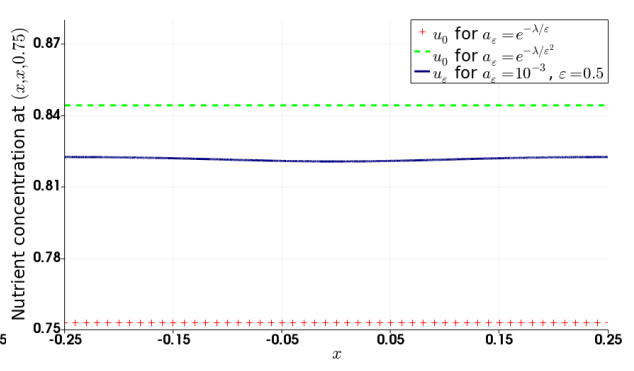

(f) $x_{3}=0.75, a_{\varepsilon}=10^{-3}$

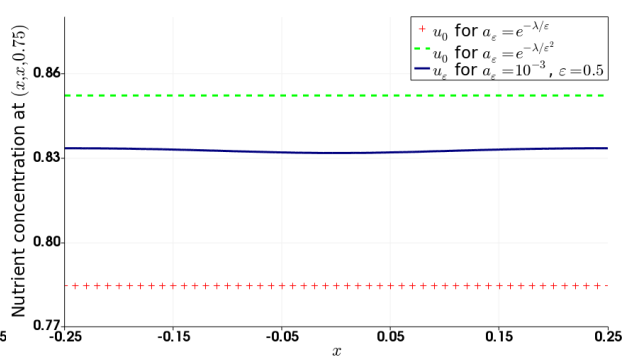

(h) $x_{3}=0.75, a_{\varepsilon}=10^{-3}, g(u)=u /(1+u)$

FIG. 3. Steady-state solutions at the root surface $\left\{x_{3}=0\right\}((\mathrm{a}),(\mathrm{c})$, and (e)) and outside the root hair zone $\left\{x_{3}=0.75\right\}$ ((b), (d), and (f)) for (2.1)-(2.3), (2.6), (2.7) (blue solid line), the problem (3.48) (red crosses), and the problem (3.57) (green dashed line), with boundary condition (5.1), $g(u)=u$, and all other parameters as in Table $1 . a_{\varepsilon}$ is decreased from $10^{-1}$ to $10^{-3}$. Subfigures $(\mathrm{g})$ and $(\mathrm{h})$ show comparisons for the nonlinear problem (with $g(u)=u /(1+u)$ ) to the problem (3.63) (green dashed line; for the full form of the continuity equation, see (3.64)) and the problem (3.52) (red crosses) using the same parameters and boundary conditions.

Copyright $@$ ㅇ by SIAM. Unauthorized reproduction of this article is prohibited. 


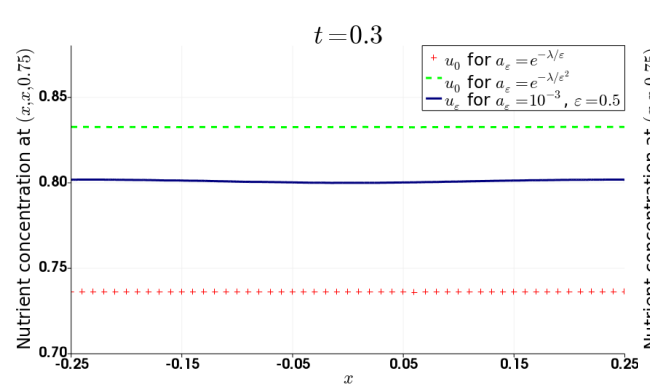

(a) $x_{3}=0.75, g(u)=u /(1+u)$

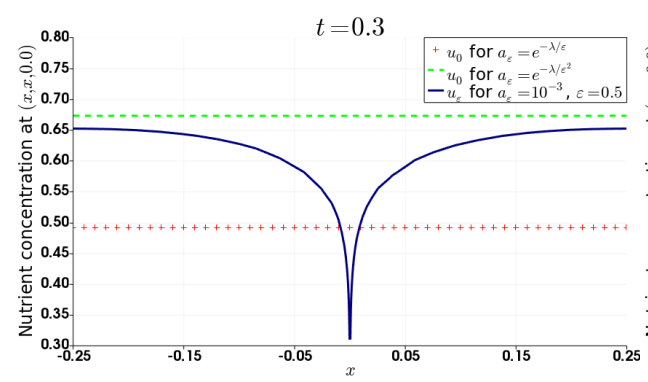

(c) $x_{3}=0.0, g(u)=u /(1+u)$

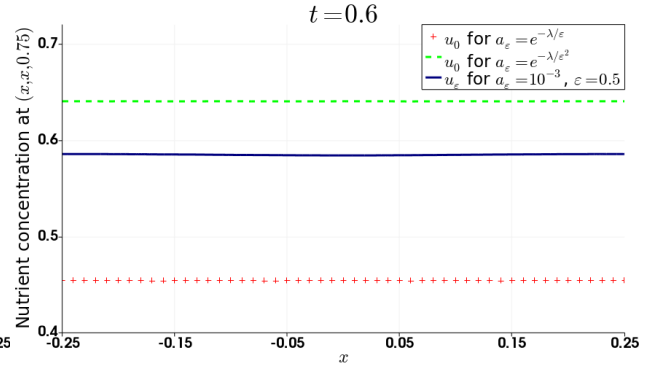

(b) $x_{3}=0.75, g(u)=u /(1+u)$

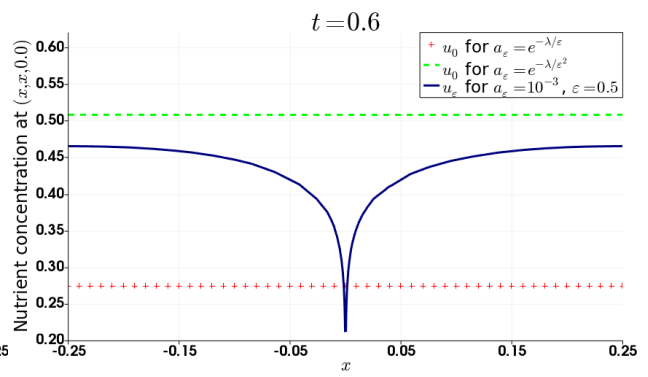

(d) $x_{3}=0.0, g(u)=u /(1+u)$

FIG. 4. Numerical solutions for (2.1)-(2.3), (2.6), (2.7) (blue solid line), the problem (3.63) (green dashed line; for the full form of the continuity equation, see (3.64)), and the problem (3.52) (red crosses), with $g(u)=u /(1+u)((\mathrm{a}),(\mathrm{b}),(\mathrm{c})$, and $(\mathrm{d}))$ and initial condition $u_{\mathrm{in}}=1$; all other parameters as in Table 1. The time derivative is discretized using the backwards Euler method, with the time step of 0.01 .

problem (3.57) overestimates, the averaged behavior of steady-state solutions of the full problem (2.1)-(2.3), (2.6), (2.7). While the solution of (3.48) provides us with a better approximation to the full-geometry behavior than that of (3.57) for $a_{\varepsilon}=10^{-1}$, the opposite is true for $a_{\varepsilon}=10^{-3}$, which confirms the validity of our asymptotic analysis results. Leading-order approximations (i.e., homogenized solutions) naturally cannot capture large depletion gradients present in full-geometry simulations near root hair surfaces. Comparison with higher-order approximations will be discussed later (see Figure 5).

Simulation results at $x_{3}=0.75$, i.e., outside the root hair zone (see Figure $3(\mathrm{~b}),(\mathrm{d}),(\mathrm{f}))$, demonstrate that as $a_{\varepsilon}$ decreases and approaches the scale relation $\varepsilon^{2} \ln \left(1 / a_{\varepsilon}\right)=O(1)$, the steady-state solution of the macroscopic model (3.57) provides a better approximation to the full model $(2.1)-(2.3),(2.6),(2.7)$ than that of (3.48).

Numerical solutions to the steady-state problem for (2.1)-(2.3), (2.6), (2.7) with a nonlinear boundary condition on $\Gamma^{\varepsilon}$, i.e., with $g\left(u_{\varepsilon}\right)=u_{\varepsilon} /\left(1+u_{\varepsilon}\right)$, and to the corresponding macroscopic problems (3.52) and (3.63) are also presented in Figure 3(g), (h). All model parameters are as in Table 1, and Picard iteration was used to solve the nonlinear problem (as described in [23]). Similar differences between solutions of the full model and the two macroscopic problems are observed in time-dependent solutions; see Figure 4. (Note that we used a zero-flux boundary condition at $x_{3}=M$ in this case, modeling competition with a neighboring root at $x_{3}=2 M$.) 


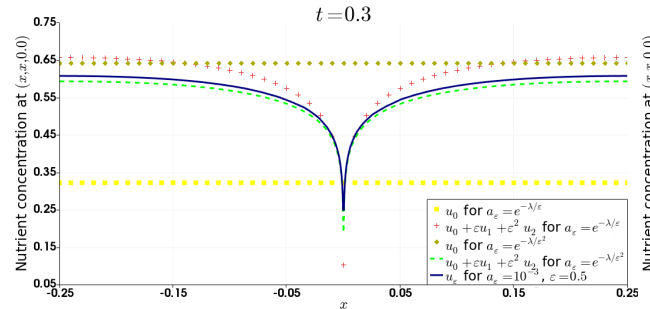

(a) $x_{3}=0.0, g(u)=u$, correctors

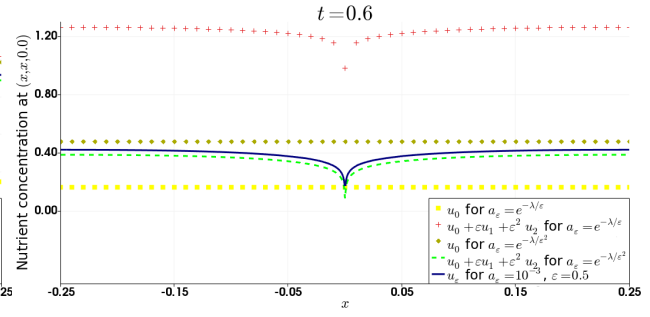

(b) $x_{3}=0.0, g(u)=u$, correctors

FIG. 5. Subfigures (a) and (b) show comparison, at the root surface $\left\{x_{3}=0\right\}$, of the linear problem (2.1)-(2.3), (2.6), (2.7) (blue solid line) with the problem (3.57) (brown diamonds), the problem (3.48) (yellow squares), the second-order approximation (3.48)-(3.51) (red crosses), and the second-order approximation (3.57)-(3.62) (green dashed line) using the same initial condition and parameters as in Figure 4.

Numerical solutions for the first and second-order corrections, given by (3.49), (3.51), (3.59), and (3.62), for the two different scale relations between $\varepsilon$ and $a_{\varepsilon}$ are presented in Figure 5.

The differences between these illustrate the importance of the correct approximation. Since we chose our parameters so that $\varepsilon^{2} \ln \left(1 / a_{\varepsilon}\right)=O(1)$, we have that solutions of (3.57)-(3.62) provide better approximations to those of the full problem (2.1)-(2.3), (2.6), (2.7) than solutions of (3.48)-(3.51).

6. Discussion. The analysis in subsection 3.1.2 using two independent small parameters $\varepsilon$ and $a$ uncovered the term $\varepsilon^{2} \ln (1 / a) u_{0,0}(t, x) \psi_{-1}^{O}$, which causes problems relating to commutation of the two limits under consideration (see (3.24)). Based on this observation, we then studied two scale relations given by $\varepsilon \ln \left(1 / a_{\varepsilon}\right)=O(1)$ and $\varepsilon^{2} \ln \left(1 / a_{\varepsilon}\right)=O(1)$. In the $\varepsilon \ln \left(1 / a_{\varepsilon}\right)=O(1)$ case, the mentioned term becomes $O(\varepsilon)$, and thus it does not affect the leading-order problem (3.48) but rather the $O(\varepsilon)$ problem (3.49). In the $\varepsilon^{2} \ln \left(1 / a_{\varepsilon}\right)=O(1)$ case, the same term becomes $O(1)$, affects the leading-order problems, and thus leads to distinguished limits; see (3.57) for the linear boundary condition and (3.63) for the nonlinear boundary condition. Notice that the sink term in the distinguished limit (3.57) is obtained by dividing the sink term in the standard limit (3.48) by $1+\lambda \kappa / D_{u}>1$, implying weaker effective nutrient uptake in the hair zone. This is because, assuming $\varepsilon^{2} \ln \left(1 / a_{\varepsilon}\right)=O(1)$, the uptake rate per unit hair surface area becomes large, causing very sharp nutrient depletion near hairs so that the diffusion is not fast enough to keep the concentration profile uniform. Under these circumstances, the difference between the nutrient concentration at the hair surface (used in the full-geometry model) and the averaged nutrient concentration (used in the sink terms) becomes significant, and this gives rise to the new limit. Subsequently, we rigorously proved the convergence of solutions of the multiscale problem to solutions of the macroscopic equations for both the linear and nonlinear boundary conditions at surfaces of root hairs and confirmed the applicability of the two limit equations (as well as higher-order correctors) in different parameter regimes via numerical simulations. 


\section{REFERENCES}

[1] G. Allaire, A. Damlamian, and U. Hornung, Two-scale convergence on periodic surfaces and applications, in Proc. International Conference Math. Modelling Flow through Porous Media, A. Bourgeat et al., eds., World Scientific, Singapore, 1996, pp. 15-25.

[2] S. BARBER, Soil Nutrient Bioavailability: A Mechanistic Approach, 2nd ed., John Wiley \& Sons, Inc., New York, 1995, http://eu.wiley.com/WileyCDA/WileyTitle/ productCd-0471587478.html.

[3] A. Bensoussan, J.-L. Lions, and G. Papanicolaou, Asymptotic Analysis of Periodic Structures, North-Holland, Amsterdam, 1978.

[4] N. Brady and R. Weil, The Nature and Properties of Soils, 11th ed., Prentice-Hall Inc., Upper Saddle River, NJ, 1996.

[5] B. Cabarrubias and P. Donato, Homogenization of some evolution problems in domains with small holes, Electron. J. Differential Equations, 2016 (2016), pp. 1-26.

[6] D. Cioranescu and F. Murat, A strange term coming from nowhere, in Topics in the Mathematical Modelling of Composite Materials, Progr. Nonlinear Differential Equations Appl. 31, A. Cherkaev and R. Kohn, eds., Birkhäuser, Boston, MA, 1997, pp. 45-93.

[7] D. Cioranescu And P. S. J. Paulin, Homogenization of Reticulated Structures, SpringerVerlag, New York, 1999.

[8] N. ClaAssen and S. Barber, A method for characterizing the relation between nutrient concentration and flux into roots of intact plants, Plant Physiol., 54 (1974), pp. 564-568.

[9] C. Conca And P. Donato, Non-homogeneous Neumann problems in domains with small holes, RAIRO Modél. Math Anal. Numér., 22 (1988), pp. 561-607.

[10] S. Datta, C. Kim, M. Pernas, N. Pires, H. Proust, T. Tam, P. Vijayakumar, and L. Dolan, Root hairs: Development, growth and evolution at the plant-soil interface, Plant Soil, 346 (2011), pp. 1-14, https://doi.org/10.1007/s11104-011-0845-4.

[11] E. Epstein ANd C. Hagen, A kinetic study of the absorption of alkali cations by barley roots, Plant Physiol., 27 (1952), pp. 457-474.

[12] L. Evans, Partial Differential Equations, American Mathematical Society, New York, 2010.

[13] E. D. Giongi and S. Spagnolo, Sulla convergenza degli integrali dell'energia per operatori ellittici del secondo ordine, Boll. Unione Mat. Ital., 8 (1973), pp. 391-411.

[14] D. Gómez, M. Lobo, M. Pérez, T. Shaposhnikova, and M. Zubova, On critical parameters in homogenization of perforated domains by thin tubes with nonlinear flux and related spectral problems, Math. Methods Appl. Sci., 38 (2015), pp. 2606-2629.

[15] W. JäGer, M. Neuss-Radu, and T. Shaposhnikova, Homogenization limit for the diffusion equation with nonlinear flux condition on the boundary of very thin holes periodically distributed in a domain, in case of a critical size, Dokl. Math., 82 (2010), pp. 736-740.

[16] W. JÄGER, M. Neuss-RAdu, And T. Shaposhnikova, Homogenization of a variational inequality for the Laplace operator with nonlinear restriction for the flux on the interior boundary of a perforated domain, Nonlinear Anal. Real World Appl., 15 (2014), pp. 367-380.

[17] J. Kevorkian and J. Cole, Multiple Scale and Singular Perturbation Methods, Appl. Math. Sci. 114, Springer-Verlag, New York, 1996.

[18] J. KöRY, Multiscale Modelling of Nutrient and Water Uptake by Plants, Ph.D. thesis, The University of Nottingham, School of Mathematical Sciences, Nottingham, UK, 2018.

[19] O. Ladyzhenskaya, V. Solonnikov, and N. Ural'Ceva, Linear and Quasilinear Equations of Parabolic Type, American Mathematical Society, New York, 1988.

[20] D. Leitner, S. Klepsch, M. Ptashnyk, A. Marchant, G. Kirk, A. Schnepf, and T. Roose, A dynamic model of nutrient uptake by root hairs, New Phytol., 185 (2010), pp. 792-802, https://doi.org/10.1111/j.1469-8137.2009.03128.x.

[21] G. Lieberman, Second Order Parabolic Differential Equations, World Scientific, Singapore, 1996.

[22] J.-L. Lions, Quelques méthodes de résolution des problèmes aux limites non linéaires, Dunod, Paris, 1969.

[23] A. Logg, K.-A. Mardal, and G. Wells, eds., Automated Solution of Differential Equations by the Finite Element Method: The FEniCS Book, Springer, 2012, http://fenicsproject. org/book/.

[24] G. D. Maso, An Introduction to Г-Convergence, Birkhäuser, Basel, 1993.

[25] F. Murat and L. Tartar, H-convergence, in Topics in the Mathematical Modelling of Composite Materials, Progr. Nonlinear Differential Equations Appl. 31, Birkhäuser, Boston, MA, 1997, pp. 21-43.

[26] M. Neuss-Radu, Some extensions of two-scale convergence, C. R. Math. Acad. Sci. Paris, 332 (1996), pp. 899-904. 
[27] G. Nguetseng, A general convergence result for a functional related to the theory of homogenization, SIAM J. Math. Anal., 20 (1989), pp. 608-623, https://doi.org/10.1137/0520043.

[28] J. Passioura, A mathematical model for the uptake of ions from the soil solution, Plant Soil, 18 (1963), pp. 225-238.

[29] M. Ptashnyk, Derivation of a macroscopic model for nutrient uptake by hairy-roots, Nonlinear Anal. Real World Appl., 11 (2010), pp. 4586-4596, https://doi.org/10.1016/j.nonrwa.2008. 10.063.

[30] M. Ptashnyk and T. Roose, Derivation of a macroscopic model for transport of strongly sorbed solutes in the soil using homogenization theory, SIAM J. Appl. Math., 70 (2010), pp. 2097-2118, https://doi.org/10.1137/080729591.

[31] R. REDLINGER, Invariant sets for strongly coupled reaction-diffusion systems under general boundary conditions, Arch. Rational Mech. Anal., 108 (1989), pp. 281-291.

[32] J. SCHÖBERL, NETGEN: An advancing front 2D/3D-mesh generator based on abstract rules, Comput. Vis. Sci., 1 (1997), pp. 41-52, https://doi.org/10.1007/s007910050004.

[33] K. Zygalakis, G. Kirk, D. Jones, M. Wissuwa, and T. Roose, A dual porosity model of nutrient uptake by root hairs, New Phytol., 192 (2011), pp. 676-688, https://doi.org/10. 1111/j.1469-8137.2011.03840.x.

Copyright (c) by SIAM. Unauthorized reproduction of this article is prohibited. 